\title{
Divergent Total Synthesis of Chaetoglines C to F
}

\author{
Yaocheng $\mathrm{Shi}^{\dagger}$, Zhi $\mathrm{Xu}^{\dagger}$, Renxiang Tan ${ }^{\ddagger}$ and Xiaoguang Lei ${ }^{*}{ }^{\dagger}$ \\ ${ }^{\dagger}$ Beijing National Laboratory for Molecular Sciences, Key Laboratory of Bioorganic Chemistry \\ and Molecular Engineering of Ministry of Education, Department of Chemical Biology, College \\ of Chemistry and Molecular Engineering, Synthetic and Functional Biomolecules Center, and \\ Peking-Tsinghua Center for Life Sciences, Peking University, Beijing 100871 \\ ‡State Key Laboratory of Pharmaceutical Biotechnology, Institute of Functional Biomolecules, \\ School of Life Sciences, Nanjing University, ${ }_{163}$ Xianlin Avenue, Nanjing 210046 \\ ${ }^{*}$ Corresponding Author: Xiaoguang Lei \\ E-mail:xglei@pku.edu.cn
}

\section{Supporting Information}

\author{
Table of Contents
}

\begin{tabular}{|l|l|}
\hline 1. Comparison of natural and synthetic chaetoglines C-F & $\mathrm{S} 2-\mathrm{S} 15$ \\
\hline 2. NMR Spectra & $\mathrm{S} 16-\mathrm{S} 23$ \\
\hline 3. Biological activity of chaetogline F & $\mathrm{S} 24$ \\
\hline 4. References & $\mathrm{S} 25$ \\
\hline
\end{tabular}




\section{Comparison of natural and synthetic chaetoglines $\mathrm{C}-\mathrm{F}$}

Table S1. Comparison of natural and synthetic chaetogline $\mathrm{C}\left({ }^{1} \mathrm{H} \mathrm{NMR}^{1}\right)$

\begin{tabular}{|c|c|c|c|}
\hline & $\begin{array}{c}\text { Natural }^{1} \\
{ }^{1} \mathrm{H} \text { NMR }(400 \mathrm{MHz}, \\
\left.\text { acetone-d }^{6}\right) \\
\delta \text { (ppm) (mult, J in Hz) }\end{array}$ & $\begin{array}{c}\text { Synthetic } \\
\left.{ }^{1} \mathrm{H} \text { NMR (400 MHz, acetone- } \mathrm{d}^{6}\right) \\
\delta(\mathrm{ppm})(\text { mult, } \mathrm{J} \text { in } \mathrm{Hz})\end{array}$ & $\begin{array}{c}\Delta \delta \\
\text { (Synthetic } \\
\text {-Natural) } \\
\text { (ppm) } \\
\end{array}$ \\
\hline 2 & $7.07(\mathrm{~s})$ & 7.08 (s) & 0.01 \\
\hline \multirow[t]{2}{*}{3} & $4.34(\mathrm{~d}, 16.1)$ & $4.31(\mathrm{~d}, 16.0)$ & -0.03 \\
\hline & $4.45(\mathrm{~d}, 16.1)$ & $4.40(\mathrm{~d}, 16.0)$ & -0.05 \\
\hline 5 & $5.39(\mathrm{dd}, 4.9,10.9)$ & 5.34 (dd, 5.0, 10.7) & -0.05 \\
\hline \multirow[t]{2}{*}{6} & $3.40(\mathrm{dd}, 10.9,15.5)$ & $3.40(\mathrm{dd}, 10.7,15.4)$ & 0 \\
\hline & 3.57 (dd, 4.9, 15.5) & 3.57 (dd, 4.8, 15.6) & 0 \\
\hline 9 & 7.67 (d, 7.7) & 7.68(d, 7.9) & 0.01 \\
\hline 10 & $7.04(t, 7.7)$ & $7.05(t, 7.6)$ & 0.01 \\
\hline 11 & $7.13(t, 7.7)$ & $7.15(t, 7.2)$ & 0.02 \\
\hline 12 & $7.26(\mathrm{~d}, 7.7)$ & $7.30(\mathrm{~d}, 8.2)$ & 0.04 \\
\hline 22 & $2.47(\mathrm{~s})$ & $2.47(\mathrm{~s})$ & 0 \\
\hline N1-Me & $3.63(\mathrm{~s})$ & $3.70(\mathrm{~s})$ & 0.07 \\
\hline
\end{tabular}

Table S2. Comparison of natural and synthetic chaetogline $\mathrm{C}\left({ }^{13} \mathrm{C}\right.$ NMR)

\begin{tabular}{|c|c|c|c|}
\hline & $\begin{array}{c}\text { Natural }^{1} \\
{ }^{13} \mathrm{C}\left\{{ }^{1} \mathrm{H}\right\} \\
\text { NMR }(101 \mathrm{MHz}, \\
\left.\text { acetone- } \mathrm{d}^{6}\right) \\
\delta(\mathrm{ppm}) \\
\end{array}$ & $\begin{array}{c}\text { Synthetic } \\
{ }^{13} \mathrm{C}\left\{{ }^{1} \mathrm{H}\right\} \text { NMR }(101 \mathrm{MHz}, \\
\left.\text { acetone- } \mathrm{d}^{6}\right) \\
\delta(\mathrm{ppm})\end{array}$ & $\begin{array}{c}\Delta \delta \\
\text { (Synthetic- } \\
\text { Natural) } \\
\text { (ppm) }\end{array}$ \\
\hline 2 & 128.6 & 128.8 & 0.2 \\
\hline 3 & 44.5 & 44.5 & 0 \\
\hline 5 & 54.8 & 55.0 & 0.2 \\
\hline 6 & 26.1 & 26.3 & 0.2 \\
\hline 7 & 110.2 & 110.2 & 0 \\
\hline 8 & 127.9 & 128.0 & 0.1 \\
\hline 9 & 119.2 & 119.3 & 0.1 \\
\hline 10 & 119.5 & 119.6 & 0.1 \\
\hline 11 & 122.2 & 122.2 & 0 \\
\hline
\end{tabular}




\begin{tabular}{|c|c|c|c|}
\hline 12 & 110.5 & 110.8 & 0.3 \\
\hline 13 & 137.9 & 137.5 & -0.4 \\
\hline 14 & 122.1 & 122.2 & 0.1 \\
\hline 15 & 121.1 & 121.4 & 0.3 \\
\hline 16 & 171.1 & 170.8 & -0.3 \\
\hline 17 & 173.3 & 173.1 & -0.2 \\
\hline 18 & 116.2 & 116.2 & 0 \\
\hline 19 & 144.9 & 144.9 & 0 \\
\hline 20 & 137.5 & 137.4 & -0.1 \\
\hline 21 & 138.4 & 138.5 & 0.1 \\
\hline 22 & 9.6 & 9.5 & -0.1 \\
\hline N1-Me & 32.6 & 32.7 & 0.1 \\
\hline \multicolumn{4}{|c|}{ Table S3. Comparison of natural and synthetic chaetogline D ( ${ }^{1} \mathrm{H}$ NMR) } \\
\hline & $\begin{array}{c}\text { Natural }^{1} \\
{ }^{1} \mathrm{H} \text { NMR }(400 \mathrm{MHz} \\
\left.\text { acetone-d } \mathrm{d}^{6}\right) \\
\delta \text { (ppm) (mult, J in Hz) }\end{array}$ & $\begin{array}{c}\text { Synthetic } \\
{ }^{1} \mathrm{H} \text { NMR }(400 \mathrm{MHz}, \\
\left.\text { acetone- } \mathrm{d}^{6}\right) \\
\delta(\mathrm{ppm})(\text { mult, } \mathrm{J} \text { in } \mathrm{Hz})\end{array}$ & $\begin{array}{c}\Delta \delta \\
\text { (Synthetic } \\
\text {-Natural) } \\
\text { (ppm) } \\
\end{array}$ \\
\hline 2 & $7.04(\mathrm{~s})$ & $7.07(\mathrm{~s})$ & 0.03 \\
\hline \multirow[t]{2}{*}{3} & $4.33(\mathrm{~d}, 16.1)$ & $4.31(\mathrm{~d}, 16.0)$ & -0.02 \\
\hline & $4.39(\mathrm{~d}, 16.1)$ & $4.38(\mathrm{~d}, 16.0)$ & -0.01 \\
\hline 5 & $5.35(\mathrm{dd}, 5.3,10.0)$ & 5.34 (dd, $5.5,10.3)$ & -0.01 \\
\hline \multirow[t]{2}{*}{6} & $3.35(\mathrm{dd}, 10.0,15.2)$ & 3.36 (dd, 10.3, 15.3) & 0.01 \\
\hline & 3.52 (dd, 5.3, 15.2) & 3.52 (dd, 5.6, 15.6) & 0 \\
\hline 9 & $7.64(\mathrm{~d}, 7.8)$ & $7.66(\mathrm{~d}, 7.9)$ & 0.02 \\
\hline 10 & $7.04(\mathrm{t}, 7.8)$ & $7.05(t, 7.4)$ & 0.01 \\
\hline 11 & $7.14(\mathrm{t}, 7.8)$ & $7.15(\mathrm{t}, 7.2)$ & 0.01 \\
\hline 12 & $7.28(\mathrm{~d}, 7.8)$ & $7.31(\mathrm{~d}, 8.2)$ & 0.03 \\
\hline 22 & $2.47(\mathrm{~s})$ & $2.47(\mathrm{~s})$ & 0 \\
\hline N1-Me & 3.67 (s) & $3.70(\mathrm{~s})$ & 0.03 \\
\hline C17-OMe & $3.68(\mathrm{~s})$ & $3.71(\mathrm{~s})$ & 0.03 \\
\hline
\end{tabular}

Table S4. Comparison of natural and synthetic chaetogline D $\left({ }^{13} \mathrm{C}\right.$ NMR $)$ 


\begin{tabular}{|c|c|c|c|}
\hline & $\begin{array}{c}\text { Natural }^{1} \\
{ }^{13} \mathrm{C}\left\{{ }^{1} \mathrm{H}\right\} \\
\text { NMR }(101 \mathrm{MHz}, \\
\left.\text { acetone-d } \mathrm{d}^{6}\right) \\
\delta(\mathrm{ppm})\end{array}$ & $\begin{array}{c}\text { Synthetic } \\
{ }^{13} \mathrm{C}\left\{{ }^{1} \mathrm{H}\right\} \text { NMR }(101 \mathrm{MHz}, \\
\left.\text { acetone-d } \mathrm{d}^{6}\right) \\
\delta(\mathrm{ppm})\end{array}$ & $\begin{array}{c}\Delta \delta \\
\text { (Syntheti } \\
\text { c- } \\
\text { Natural) } \\
\text { (ppm) } \\
\end{array}$ \\
\hline 2 & 128.0 & 128.8 & 0.2 \\
\hline 3 & 44.5 & 44.4 & -0.1 \\
\hline 5 & 55.0 & 54.9 & -0.1 \\
\hline 6 & 26.1 & 26.4 & 0.3 \\
\hline 7 & 110.4 & 110.5 & 0.1 \\
\hline 8 & 128.7 & 128.7 & 0 \\
\hline 9 & 119.2 & 119.2 & 0 \\
\hline 10 & 119.6 & 119.6 & 0 \\
\hline 11 & 122.3 & 122.3 & 0 \\
\hline 12 & 110.2 & 110.3 & 0.1 \\
\hline 13 & 138.0 & 138.0 & 0 \\
\hline 14 & 122.2 & 122.2 & 0 \\
\hline 15 & 121.2 & 121.3 & 0.1 \\
\hline 16 & 170.7 & 170.5 & 0 \\
\hline 17 & 172.6 & 172.6 & 0 \\
\hline 18 & 116.3 & 116.3 & 0 \\
\hline 19 & 145.0 & 144.9 & -0.1 \\
\hline 20 & 137.6 & 137.6 & 0 \\
\hline 21 & 138.5 & 138.5 & 0 \\
\hline 22 & 9.6 & 9.5 & -0.1 \\
\hline N1-Me & 32.7 & 32.7 & 0 \\
\hline C17-OMe & 52.4 & 52.4 & 0 \\
\hline
\end{tabular}

Table S5. Comparison of natural and synthetic chaetogline E ( ${ }^{1} \mathrm{H}$ NMR)

\begin{tabular}{|c|c|c|}
\hline 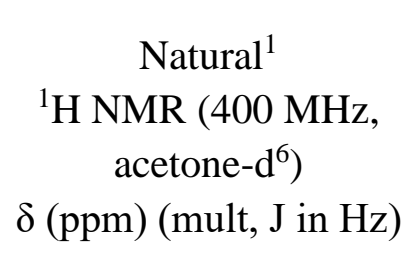 & $\begin{array}{c}\text { Synthetic } \\
{ }^{1} \mathrm{H} \text { NMR }(400 \mathrm{MHz}, \\
\left.\text { acetone- } \mathrm{d}^{6}\right) \\
\delta(\mathrm{ppm})(\text { mult, } \mathrm{J} \text { in } \mathrm{Hz})\end{array}$ & $\begin{array}{c}\Delta \delta \\
\text { (Syntheti } \\
\text { c- } \\
\text { Natural) } \\
\text { (ppm) }\end{array}$ \\
\hline
\end{tabular}




\begin{tabular}{cccc}
3 & $6.22(\mathrm{~s})$ & $6.21(\mathrm{~s})$ & -0.01 \\
5 & $5.55(\mathrm{~d}, 5.6)$ & $5.55(\mathrm{~d}, 5.2)$ & 0 \\
6 & $3.10(\mathrm{dd}, 5.6,15.3)$ & $3.09(\mathrm{dd}, 6.3,15.4)$ & -0.01 \\
& $3.40(\mathrm{~d}, 15.3)$ & $3.40(\mathrm{~d}, 15.4)$ & 0 \\
9 & $7.47(\mathrm{~d}, 7.8)$ & $7.49(\mathrm{~d}, 7.9)$ & 0.02 \\
10 & $7.03(\mathrm{t}, 7.8)$ & $7.03(\mathrm{t}, 7.2)$ & 0 \\
11 & $7.15(\mathrm{t}, 7.8)$ & $7.16(\mathrm{t}, 7.2)$ & 0.01 \\
12 & $7.33(\mathrm{~d}, 7.8)$ & $7.36(\mathrm{~d}, 8.2)$ & 0.03 \\
22 & $2.55(\mathrm{~s})$ & $2.56(\mathrm{~s})$ & 0.01 \\
$\mathrm{~N} 1-\mathrm{Me}$ & $4.01(\mathrm{~s})$ & $4.05(\mathrm{~s})$ & 0.04 \\
\hline
\end{tabular}

Table S6. Comparison of natural and synthetic chaetogline E $\left({ }^{13} \mathrm{C} \mathrm{NMR}\right)$

\begin{tabular}{|c|c|c|c|}
\hline & $\begin{array}{c}\text { Natural }^{1} \\
{ }^{13} \mathrm{C}\left\{{ }^{1} \mathrm{H}\right\} \\
\text { NMR }(101 \mathrm{MHz}, \\
\left.\text { acetone-d }{ }^{6}\right) \\
\delta(\mathrm{ppm})\end{array}$ & $\begin{array}{c}\text { Synthetic } \\
{ }^{13} \mathrm{C}\left\{{ }^{1} \mathrm{H}\right\} \text { NMR }(101 \mathrm{MHz}, \\
\left.\text { acetone- } \mathrm{d}^{6}\right) \\
\delta(\mathrm{ppm})\end{array}$ & $\begin{array}{c}\Delta \delta \\
\text { (Synthetic } \\
\text {-Natural) } \\
\text { (ppm) }\end{array}$ \\
\hline 2 & 134.8 & 134.9 & 0.1 \\
\hline 3 & 55.0 & 55.0 & 0 \\
\hline 5 & 52.8 & 52.8 & 0 \\
\hline 6 & 25.5 & 25.6 & 0.1 \\
\hline 7 & 108.5 & 108.6 & 0.1 \\
\hline 8 & 127.7 & 127.8 & 0.1 \\
\hline 9 & 118.9 & 119.0 & 0.1 \\
\hline 10 & 120.0 & 120.1 & 0.1 \\
\hline 11 & 122.7 & 122.7 & 0 \\
\hline 12 & 110.5 & 110.6 & 0.1 \\
\hline 13 & 139.9 & 140.0 & 0.1 \\
\hline 14 & 124.9 & 125.1 & 0.2 \\
\hline 15 & 121.1 & 121.3 & 0.2 \\
\hline 16 & 172.1 & 171.8 & -0.3 \\
\hline 17 & 173.2 & 172.8 & -0.5 \\
\hline 18 & 117.2 & 117.2 & 0 \\
\hline
\end{tabular}




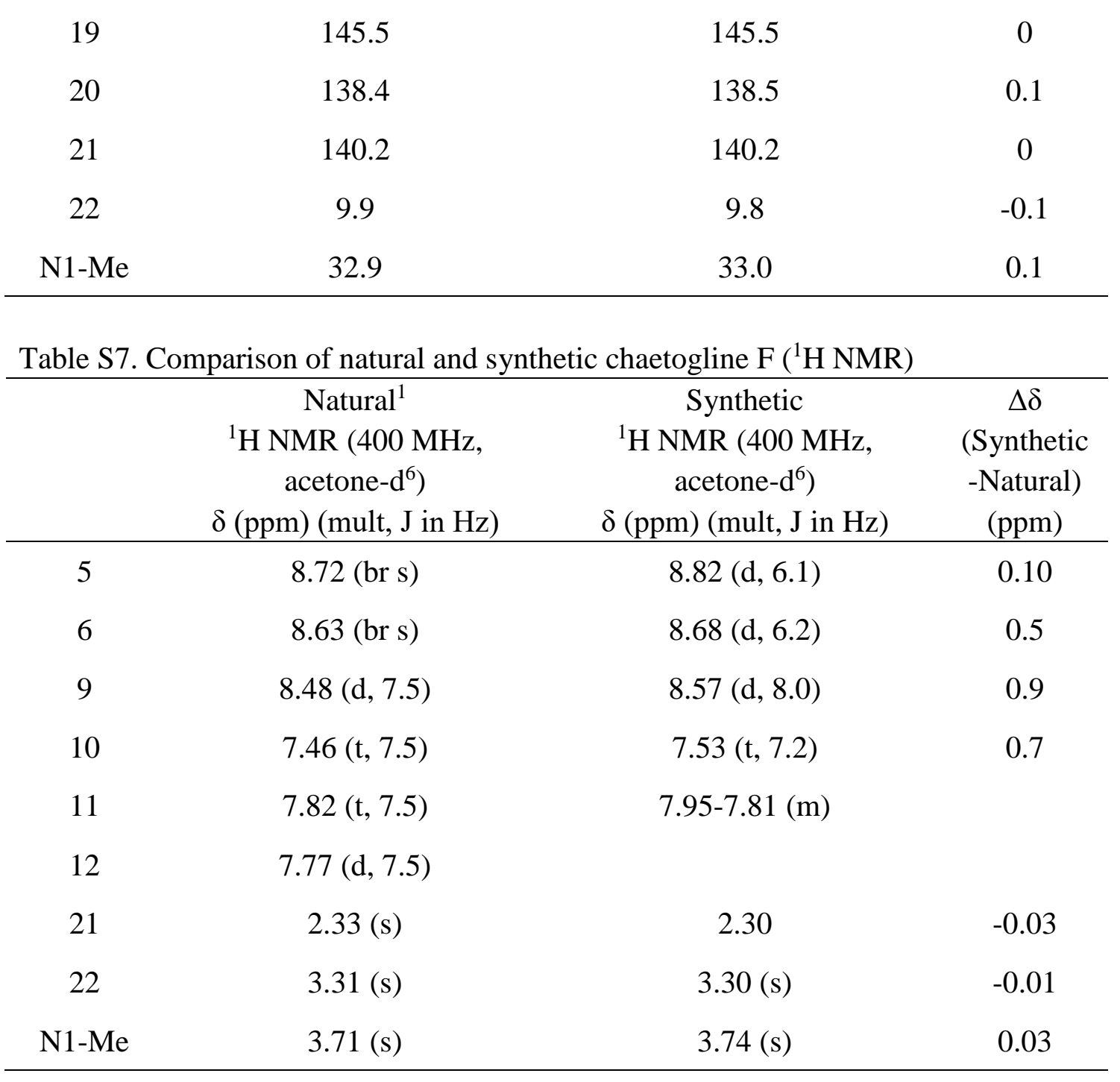

Table S8. Comparison of natural and synthetic chaetogline F $\left({ }^{13} \mathrm{C}\right.$ NMR)

\begin{tabular}{cccc}
\hline & $\begin{array}{c}\text { Natural } \\
\text { NMR }(101 \mathrm{MHz}, \\
\left.\text { acetone- } \mathrm{d}^{6}\right) \\
\delta(\mathrm{ppm})\end{array}$ & $\begin{array}{c}\text { Synthetic } \\
{ }^{13} \mathrm{C}\left\{{ }^{13} \mathrm{C}{ }^{1} \mathrm{H}\right\} \\
\text { NMR }(101 \mathrm{MHz}, \\
\left.\text { acetone- } \mathrm{d}^{6}\right) \\
\delta(\mathrm{ppm})\end{array}$ & $\begin{array}{c}\Delta \delta \\
(\text { Synthetic } \\
\text {-Natural }) \\
(\mathrm{ppm})\end{array}$ \\
\hline 2 & 135.9 & 135.9 & 0 \\
3 & 137.7 & 137.5 & -0.2 \\
5 & 129.8 & 129.6 & -0.2 \\
6 & 116.9 & 117.1 & 0.2 \\
7 & 134.6 & 134.9 & 0.3 \\
8 & 120.3 & 120.4 & 0.1 \\
9 & 124.0 & 124.2 & 0.2 \\
10 & 122.7 & 122.9 & 0.2
\end{tabular}




\begin{tabular}{cccc}
11 & 132.8 & 133.1 & 0.3 \\
12 & 111.8 & 112.0 & 0.2 \\
13 & 146.0 & 146.3 & 0.3 \\
14 & 109.9 & 109.1 & 0.8 \\
15 & 125.6 & 125.7 & 0.1 \\
16 & 118.5 & 118.7 & 0.2 \\
17 & 149.1 & 148.7 & -0.4 \\
18 & 136.6 & 136.7 & 0.1 \\
19 & 144.9 & 144.3 & -0.6 \\
20 & 168.4 & 168.2 & -0.2 \\
21 & 13.2 & 13.2 & 0 \\
22 & 52.1 & 52.2 & 0.1 \\
$\mathrm{~N} 1-\mathrm{Me}$ & 31.3 & 31.5 & 0.2 \\
\hline
\end{tabular}

Table S9. $[\alpha]_{D}$ Comparison of natural and synthetic chaetoglines C-E.

\begin{tabular}{lll}
\hline & Natural $^{1}$ & Synthetic \\
\hline Chaetogline C & {$[\alpha]^{20}{ }_{\mathrm{D}}=-24.0(\mathrm{c} 0.21, \mathrm{MeOH})$} & {$[\alpha]^{29}{ }_{\mathrm{D}}=-30.2^{\circ}(\mathrm{c} 0.1, \mathrm{MeOH})$} \\
Chaetogline D & {$[\alpha]^{20}{ }_{\mathrm{D}}=-32.9^{\circ}(\mathrm{c} 0.17, \mathrm{MeOH})$} & {$[\alpha]^{29}{ }_{\mathrm{D}}=-39.0^{\circ}(\mathrm{c} 0.1, \mathrm{MeOH})$} \\
Chaetogline E & {$[\alpha]^{20}{ }_{\mathrm{D}}=+25.5^{\circ}(\mathrm{c} 0.13, \mathrm{MeOH})$} & {$[\alpha]^{29}{ }_{\mathrm{D}}=+33.5^{\circ}(\mathrm{c} 0.1, \mathrm{MeOH})$} \\
Chaetogline F & No reported data & {$[\alpha]^{25}{ }_{\mathrm{D}}=+90.1^{\circ}(\mathrm{c} 0.1, \mathrm{MeOH})$}
\end{tabular}


NMR spectra of synthetic chaetoglines C-F and natural chaetoglines C-F.

${ }^{1} \mathrm{H}$ NMR of synthetic chaetogline C (3)

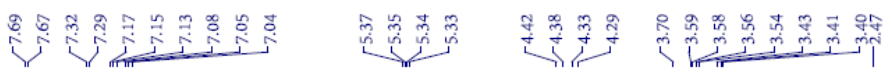

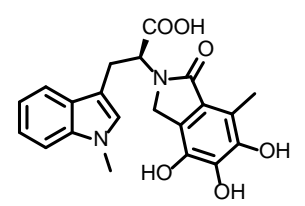

chaetogline $C(3)$

${ }^{1} \mathrm{H}$ NMR $\left(400 \mathrm{MHz}\right.$, acetone- $\mathrm{d}^{6}$, r.t.)

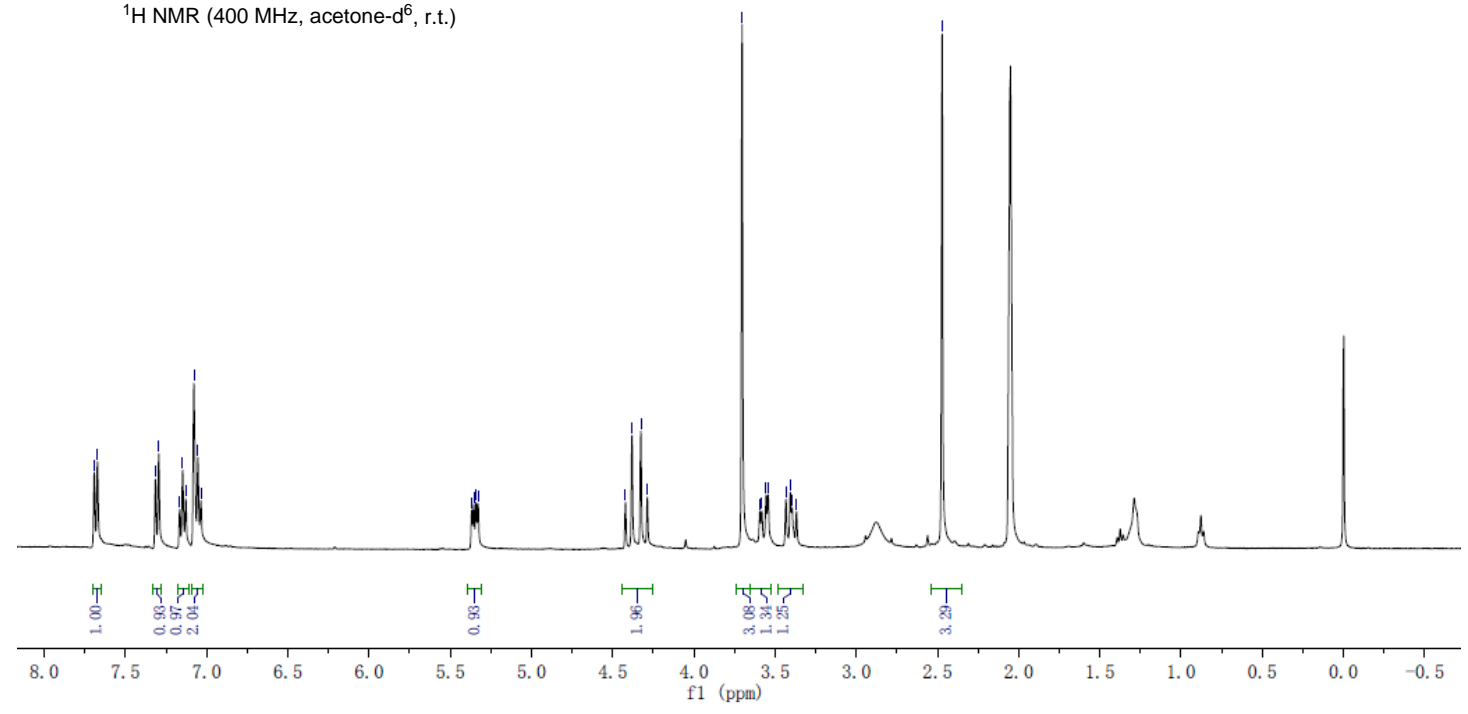

${ }^{1} \mathrm{H}$ NMR of natural chaetogline C (3) ${ }^{1}$

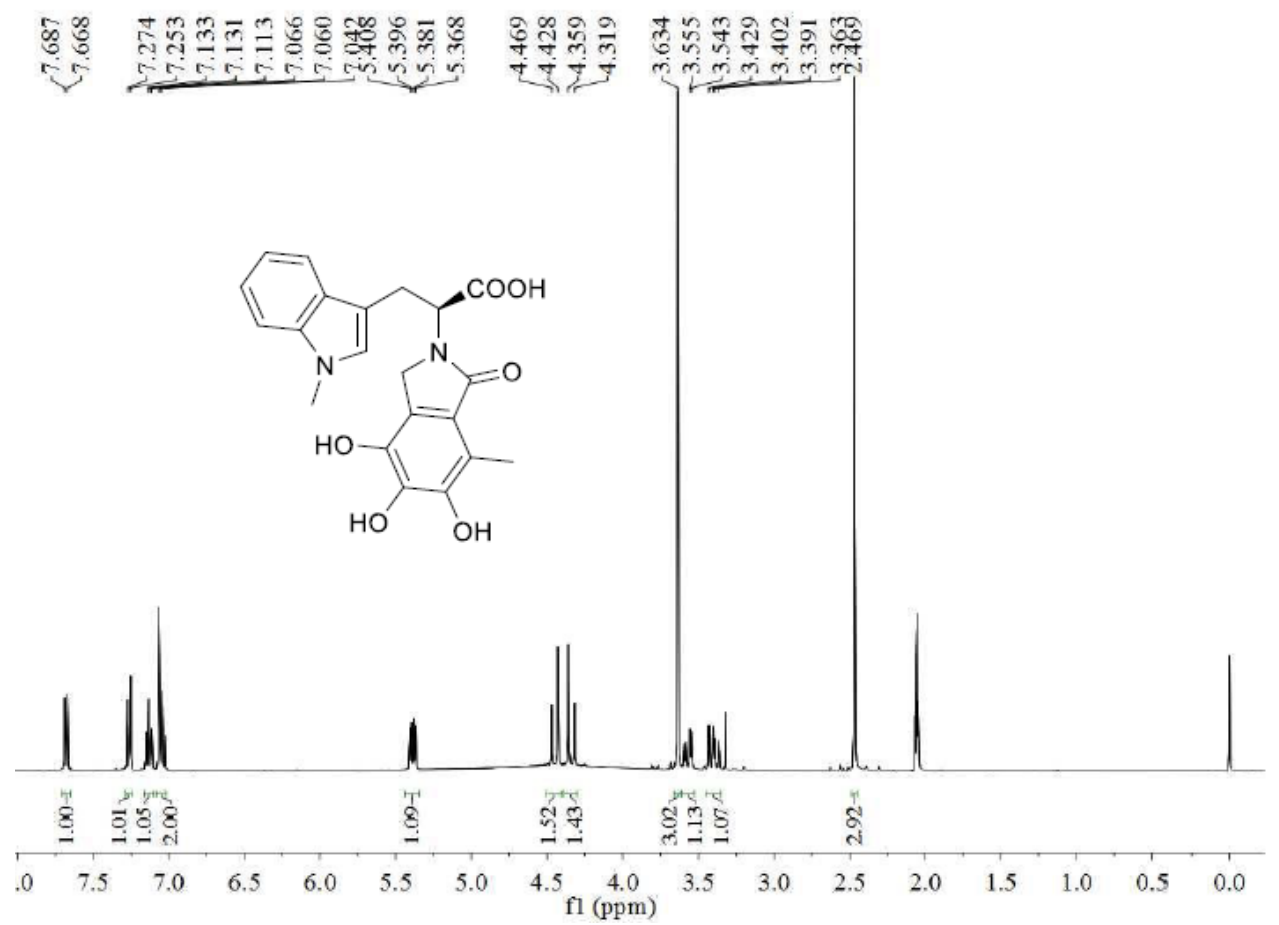


${ }^{13} \mathrm{C}\left\{{ }^{1} \mathrm{H}\right\}$ NMR of synthetic chaetogline C (3)
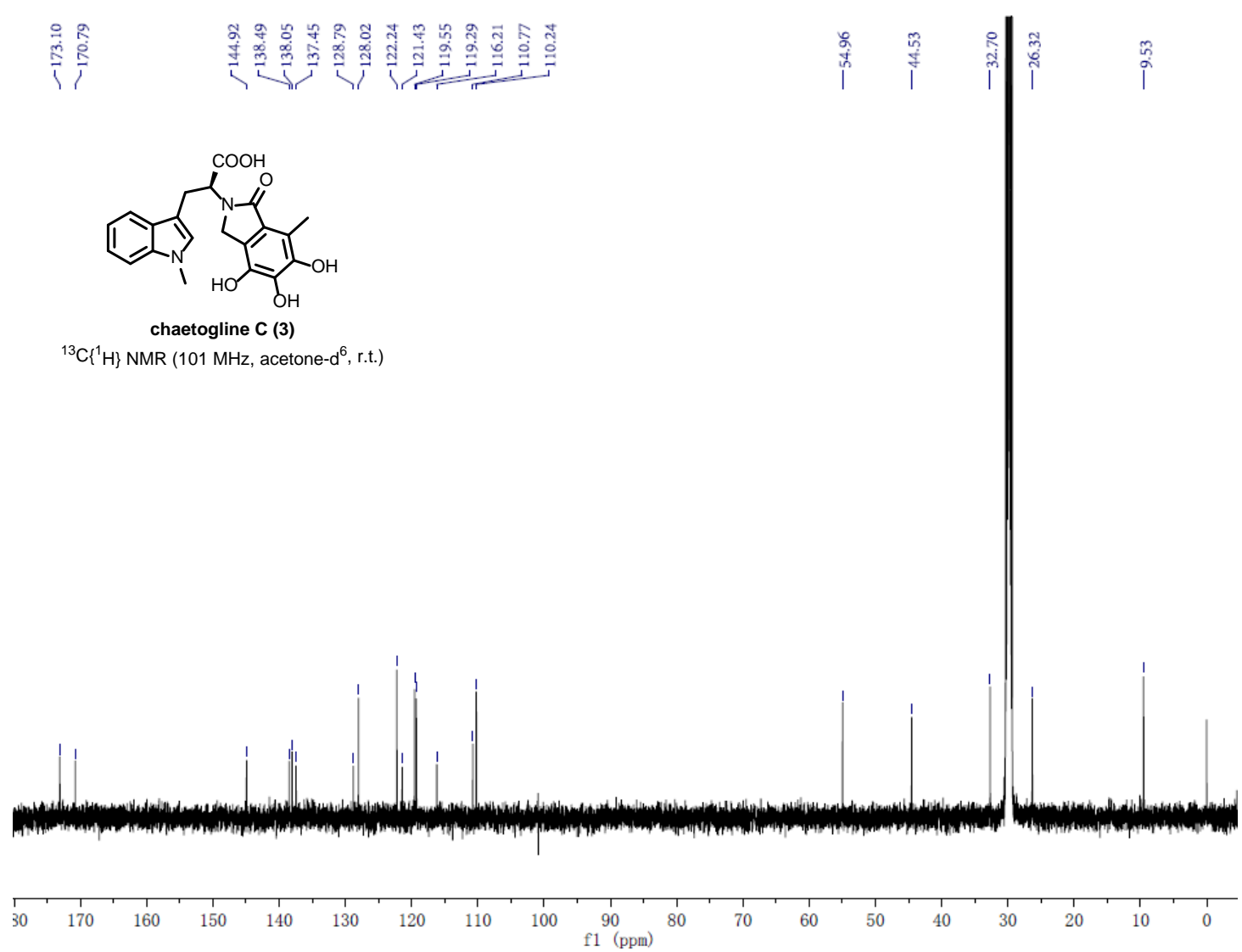

${ }^{13} \mathrm{C}\left\{{ }^{1} \mathrm{H}\right\}$ NMR of natural chaetogline $\mathrm{C}(3)^{1}$

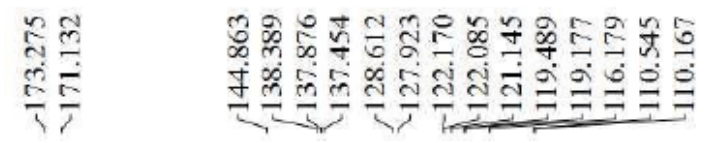

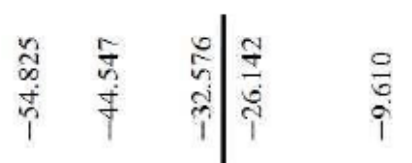<smiles>Cc1c(O)c(O)c(O)c2c1C(=O)N(C(Cc1cn(C)c3ccccc13)C(=O)O)C2</smiles>

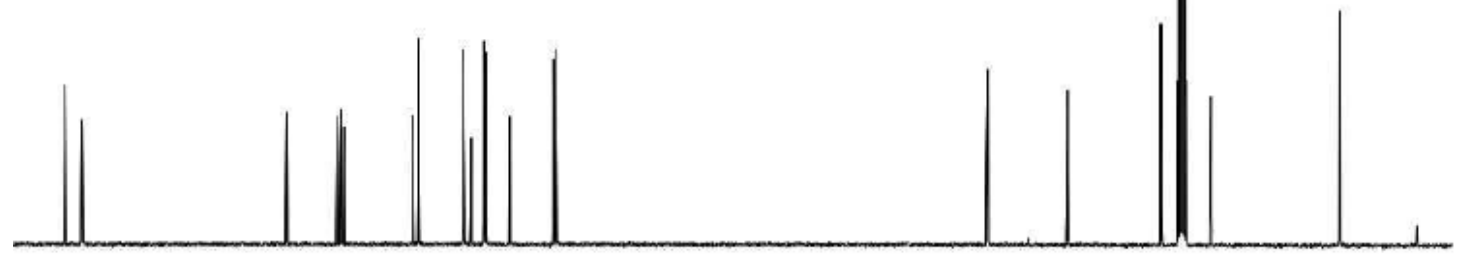

$\begin{array}{rrrrrrrrrrrrrrrrrrrrrrr}30 & 170 & 160 & 150 & 140 & 130 & 120 & 110 & 100 \underset{(\mathrm{ppm})}{90} & 80 & 70 & 60 & 50 & 40 & 30 & 20 & 10 & 0\end{array}$ 
${ }^{1} \mathrm{H}$ NMR of synthetic chaetogline D (4)

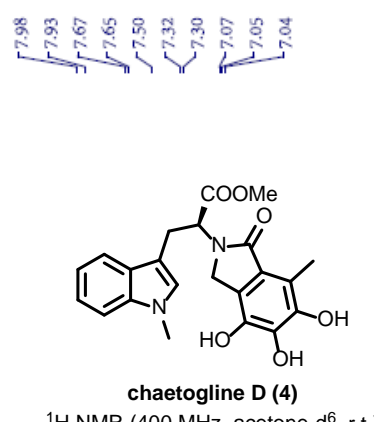

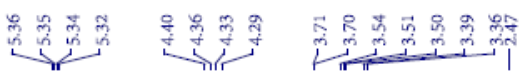

1H NMR (400 MHz, acetone-d ${ }^{6}$, r.t.)

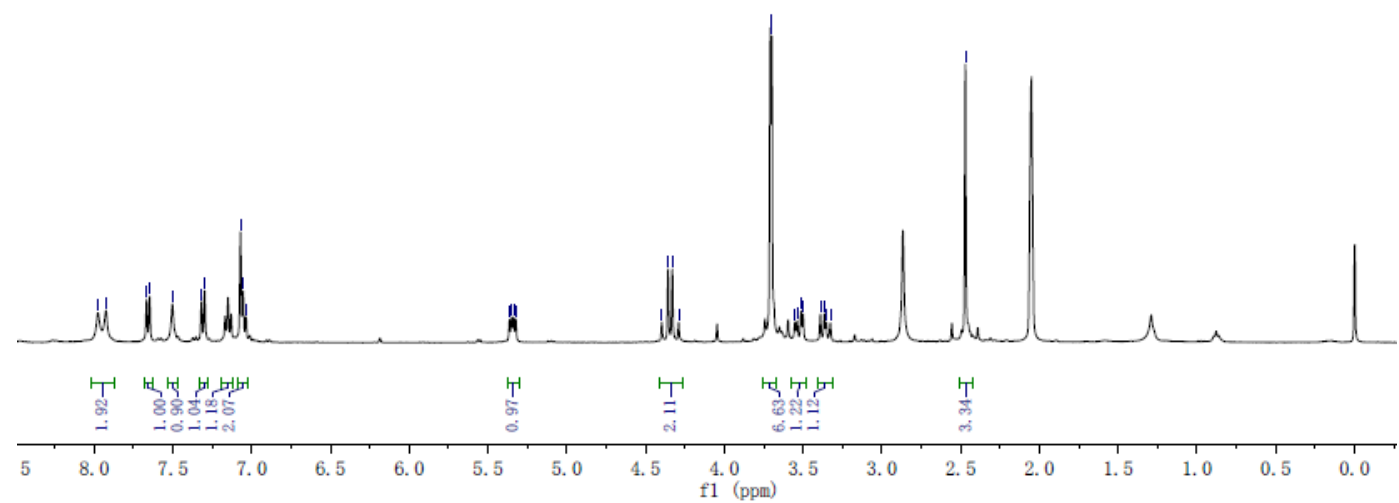

${ }^{1} \mathrm{H}$ NMR of natural chaetogline D (4) ${ }^{1}$

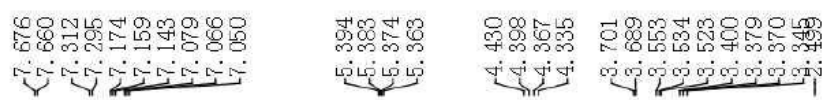

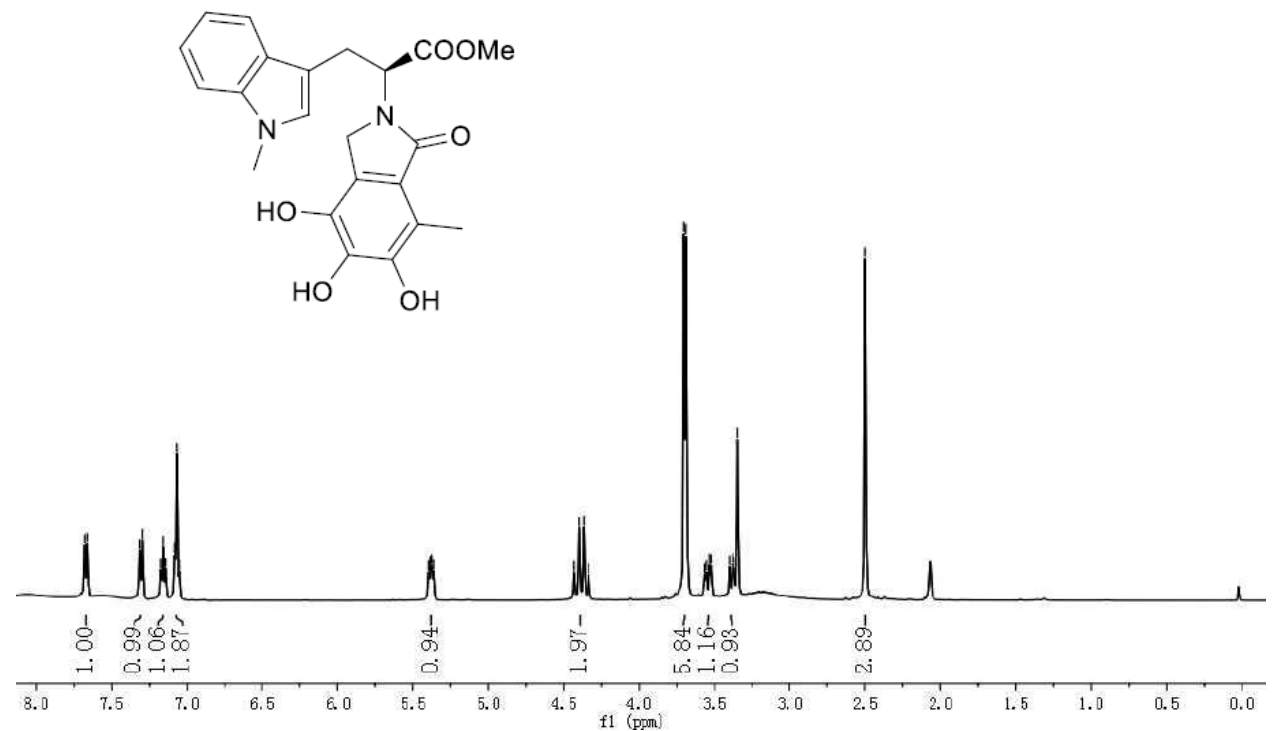


${ }^{13} \mathrm{C}\left\{{ }^{1} \mathrm{H}\right\}$ NMR of synthetic chaetogline D (4)
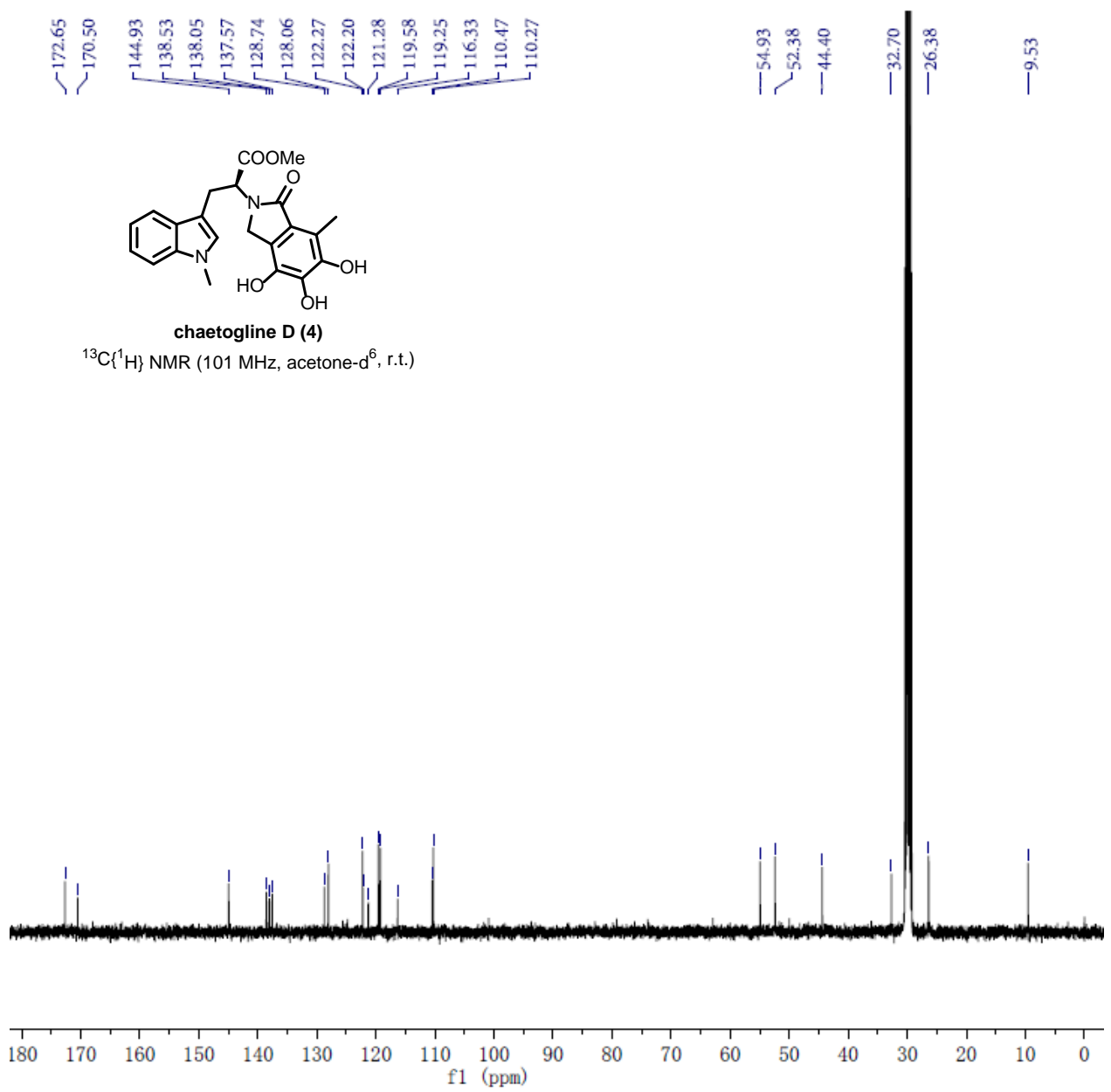

${ }^{13} \mathrm{C}\left\{{ }^{1} \mathrm{H}\right\}$ NMR of natural chaetogline D (4) ${ }^{1}$
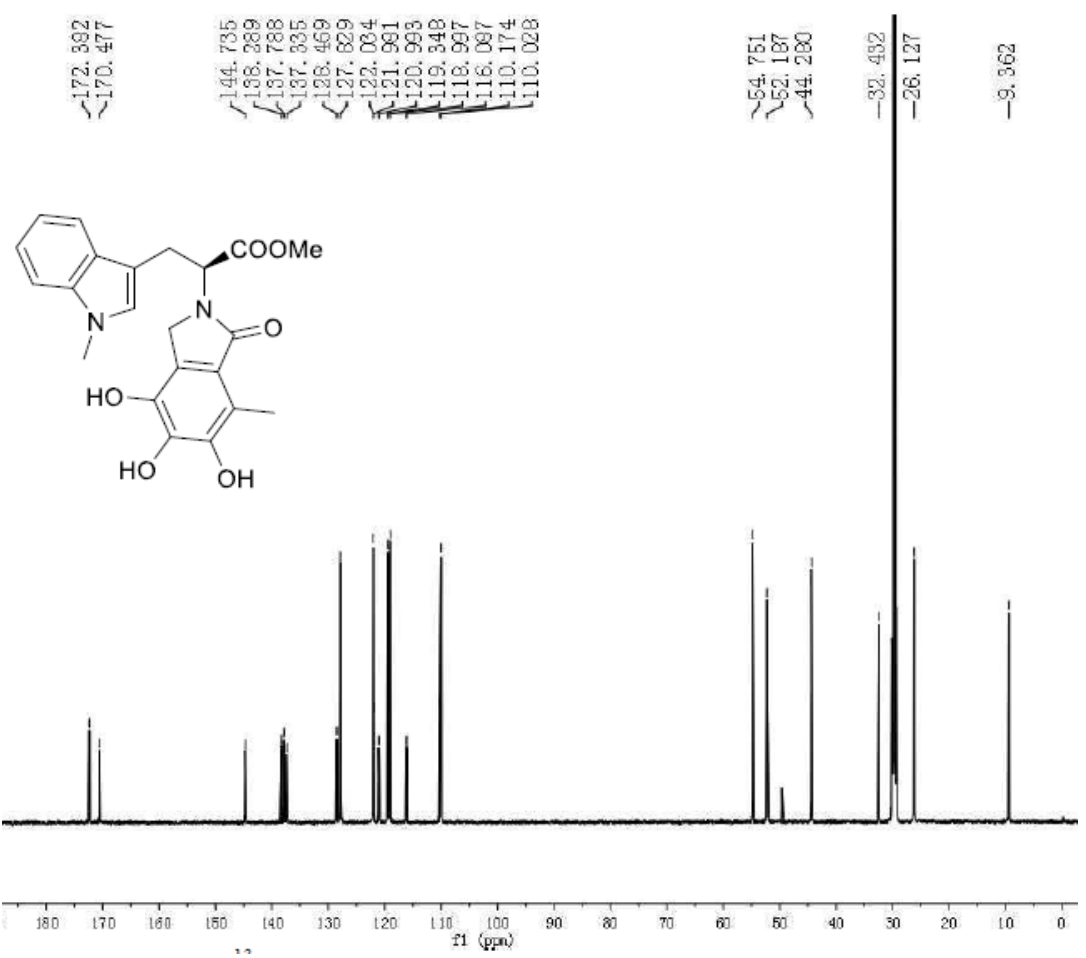
${ }^{1} \mathrm{H}$ NMR of synthetic chaetogline E (5)

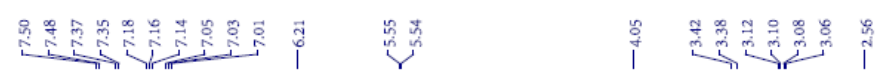

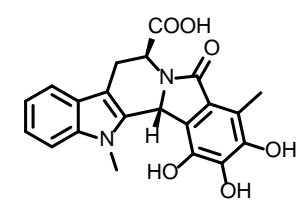

chaetogline $\mathrm{E}$ (5)

${ }^{1}{ }_{H}$ NMR (400 MHz, acetone-d ${ }^{6}$, r.t.)

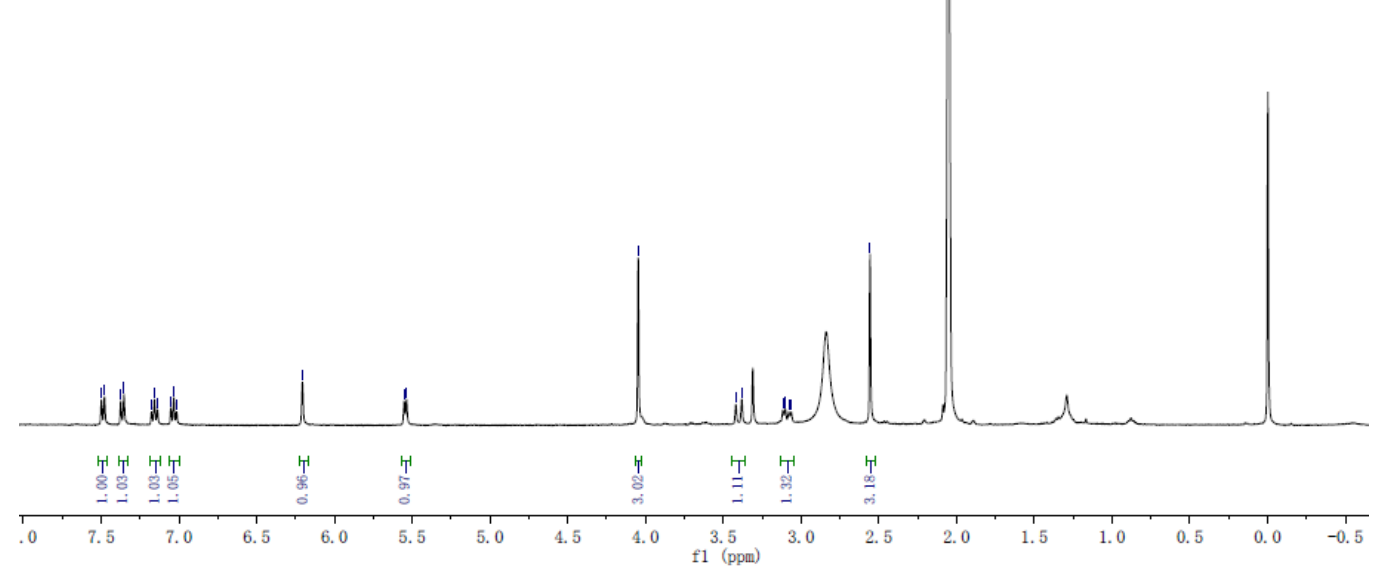

${ }^{1} \mathrm{H}$ NMR of natural chaetogline $\mathrm{E}(\mathbf{5})^{1}$

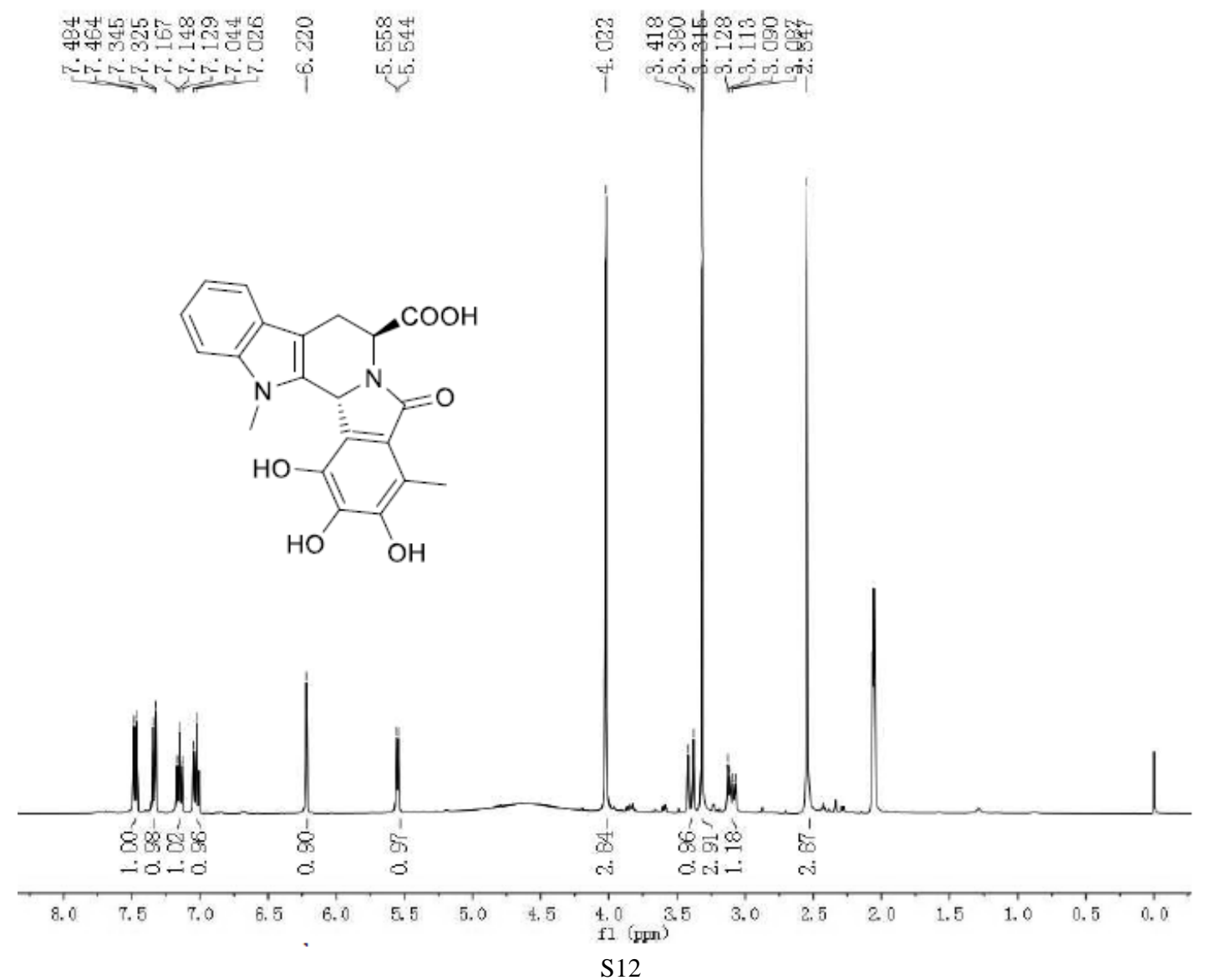


${ }^{13} \mathrm{C}\left\{{ }^{1} \mathrm{H}\right\}$ NMR of synthetic chaetogline $\mathrm{E}$ (5)
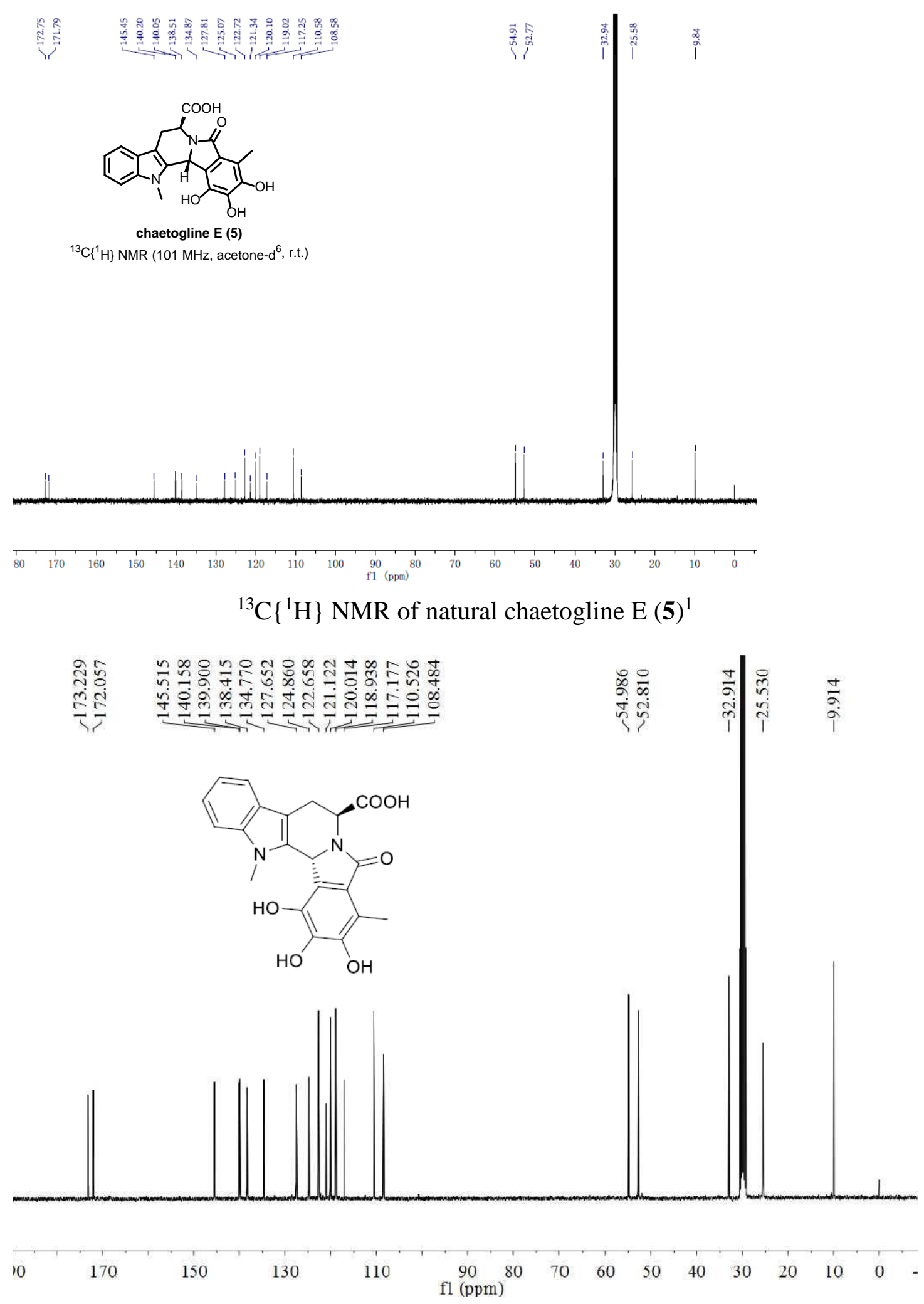
${ }^{1} \mathrm{H}$ NMR of synthetic chaetogline F (6)
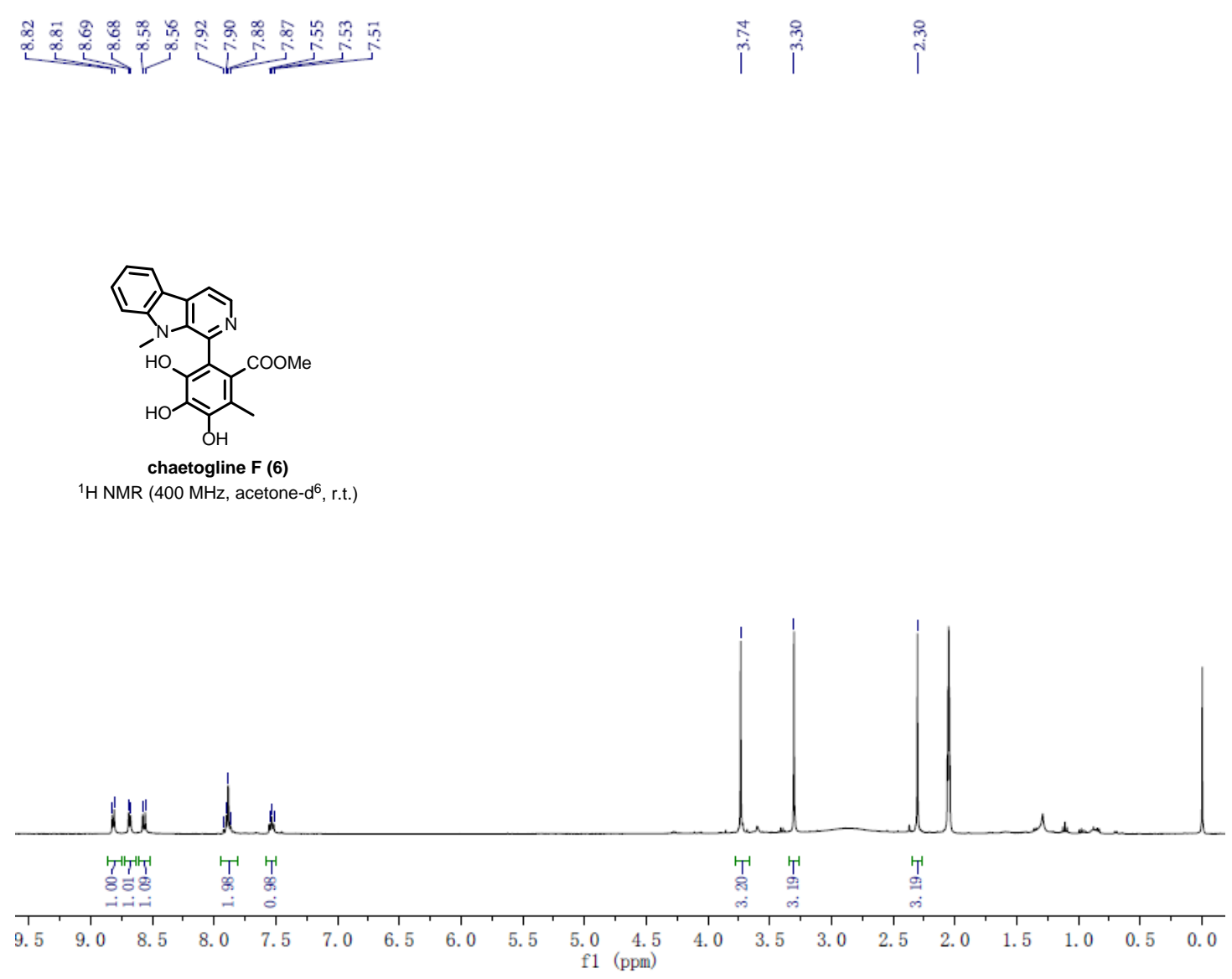

${ }^{1} \mathrm{H}$ NMR of natural chaetogline $\mathrm{F}(\mathbf{6})^{1}$

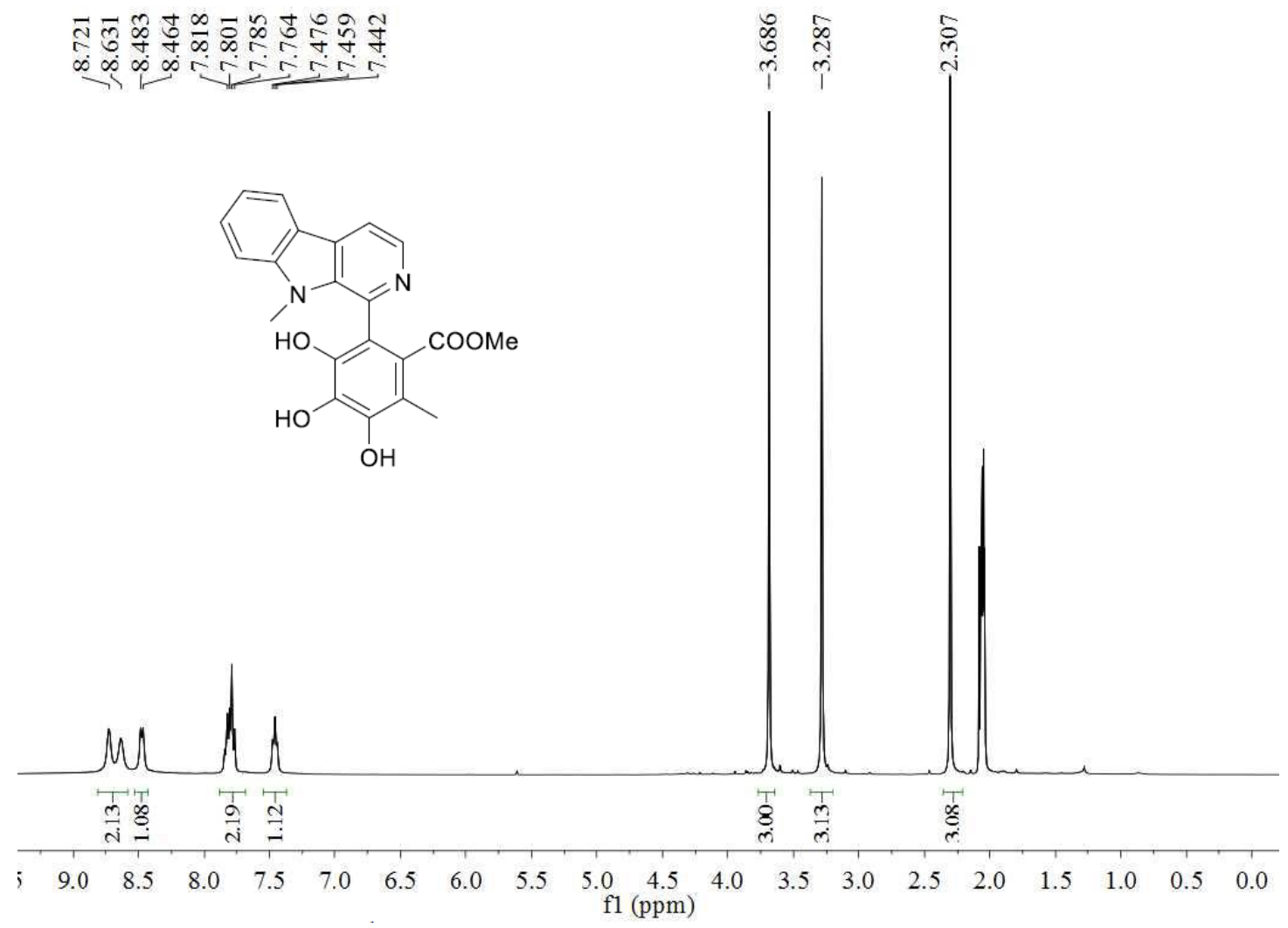


${ }^{13} \mathrm{C}\left\{{ }^{1} \mathrm{H}\right\}$ NMR of synthetic chaetogline F (6)

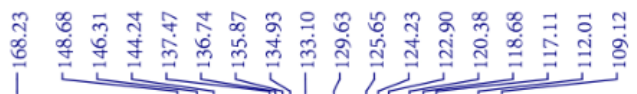

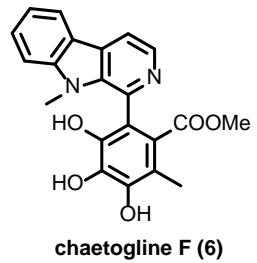

${ }^{13} \mathrm{C}\left\{{ }^{1} \mathrm{H}\right\}$ NMR (101 MHz, acetone-d $\mathrm{d}^{6}$, r.t.)
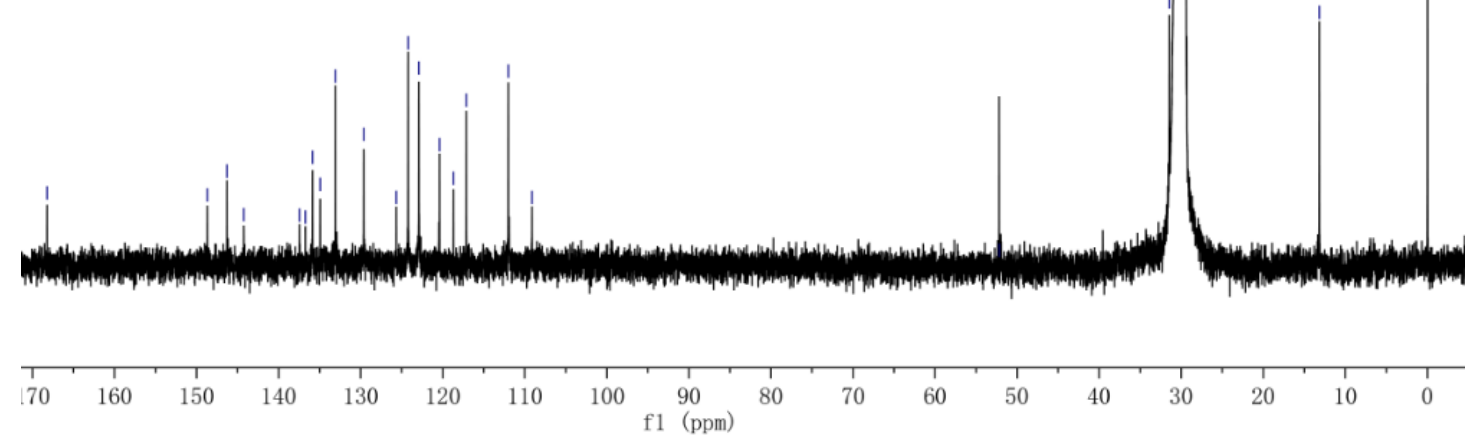

${ }^{13} \mathrm{C}\left\{{ }^{1} \mathrm{H}\right\}$ NMR of natural chaetogline $\mathrm{F}(\mathbf{6})^{1}$

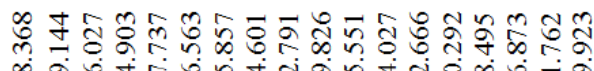

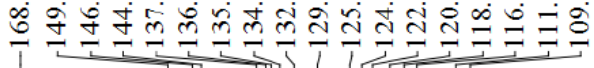
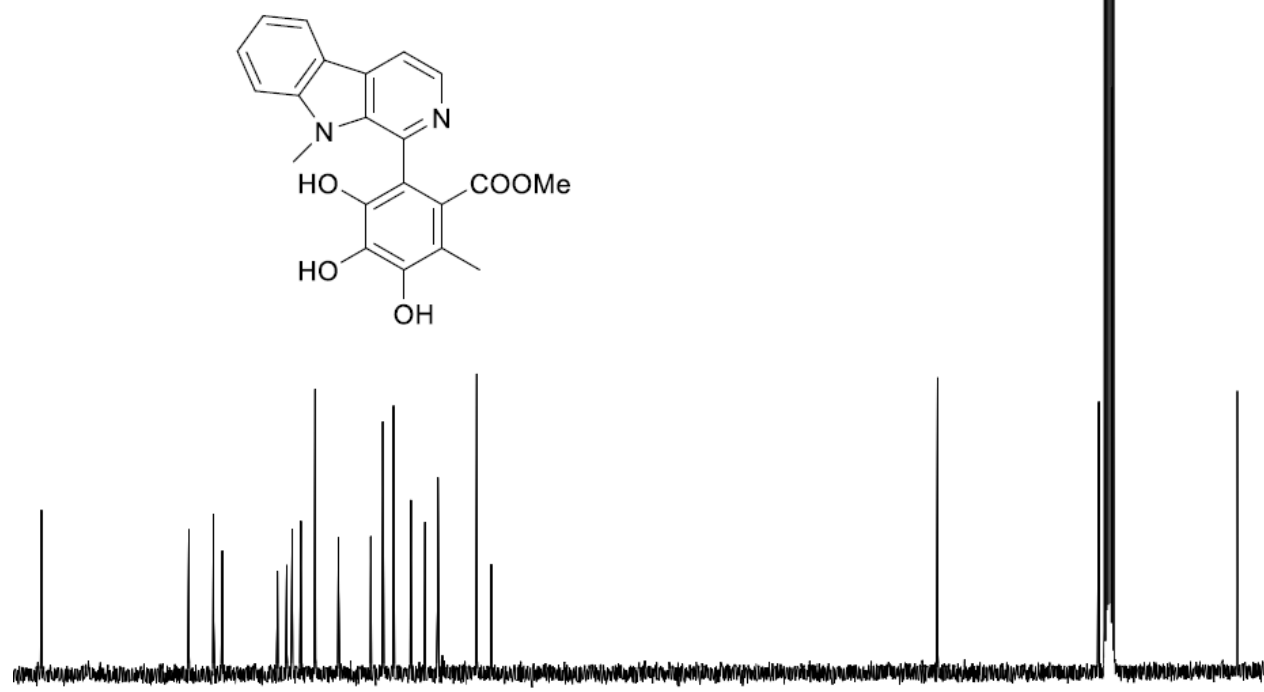

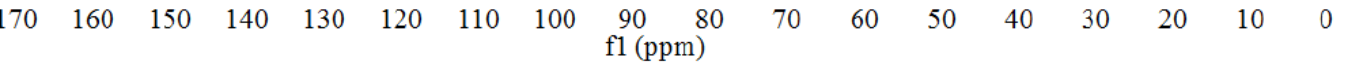




\section{NMR spectra}
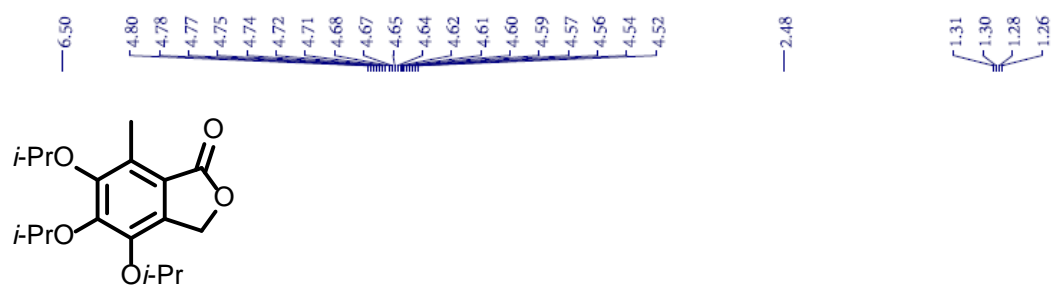

10

${ }^{1} \mathrm{H}$ NMR $\left(400 \mathrm{MHz}, \mathrm{CDCl}_{3}\right.$, r.t. $)$

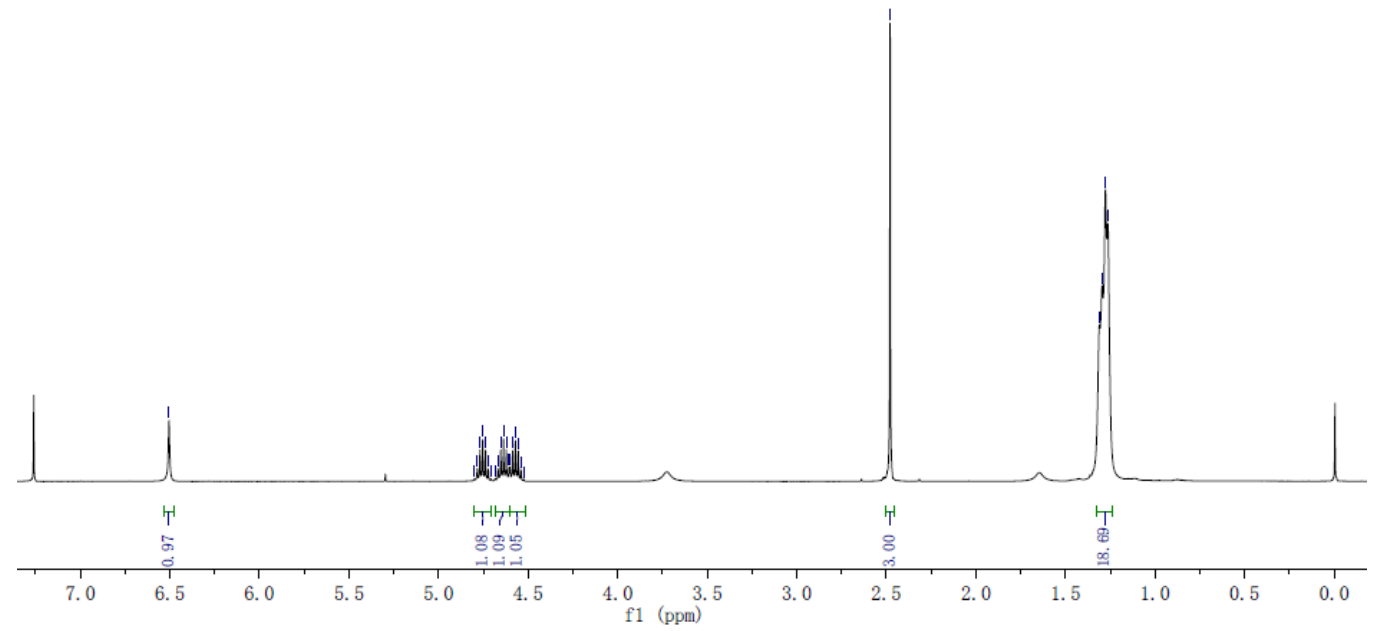

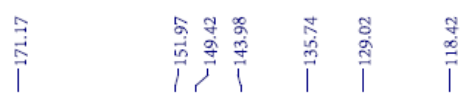

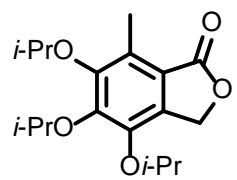

10

${ }^{13} \mathrm{C}\left\{{ }^{1} \mathrm{H}\right\}$ NMR $\left(101 \mathrm{MHz}, \mathrm{CDCl}_{3}\right.$, r.t. $)$

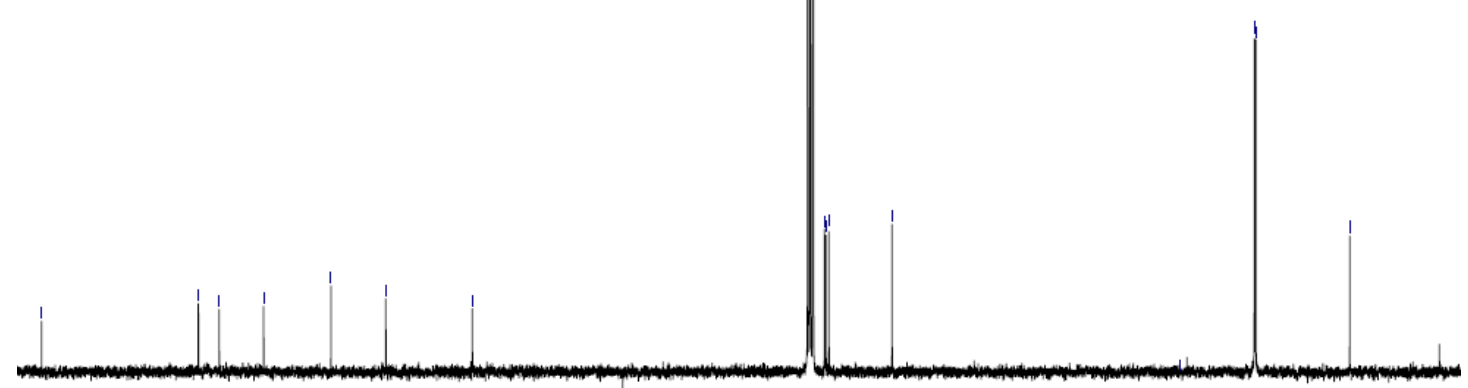

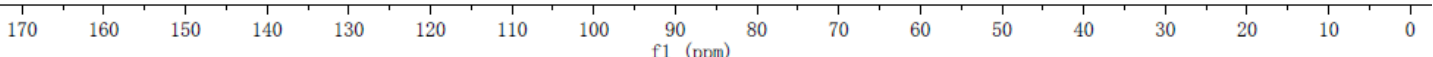




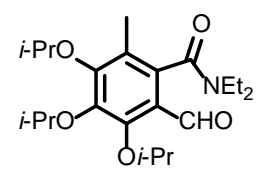

s-1

${ }^{1} \mathrm{H}$ NMR $\left(400 \mathrm{MHz}, \mathrm{CDCl}_{3}\right.$, r.t. $)$

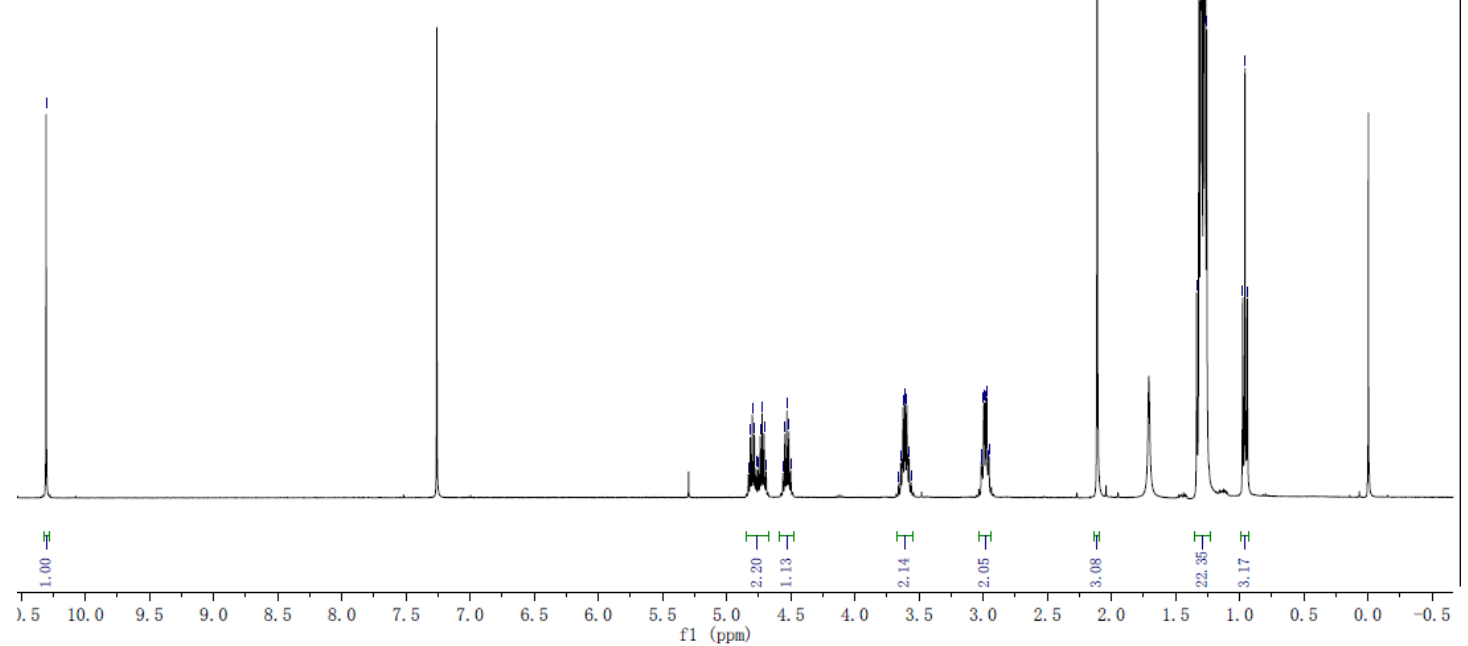

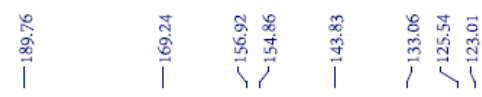<smiles>CCCOc1c(C=O)c(C(=O)NCC)c(C)c(OC(C)C)c1OCCC</smiles>

s-1

${ }^{13} \mathrm{C}\left\{{ }^{1} \mathrm{H}\right\}$ NMR $\left(101 \mathrm{MHz}, \mathrm{CDCl}_{3}\right.$, r.t. $)$

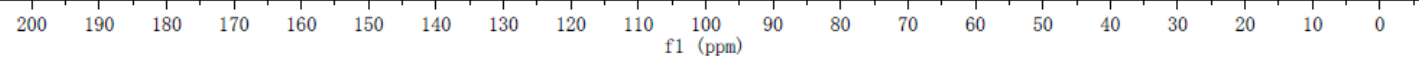




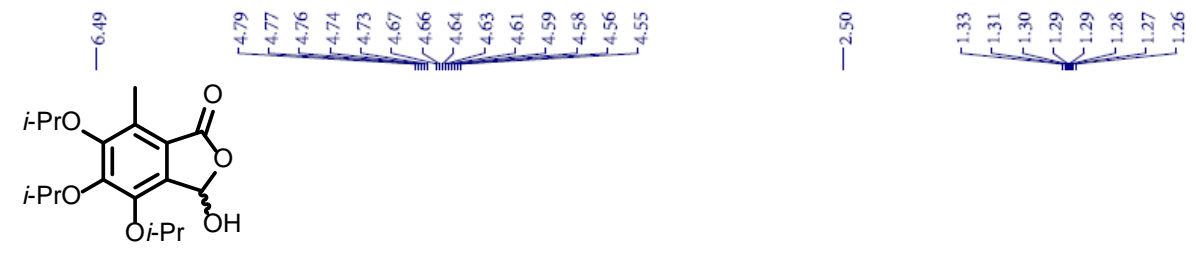

11

${ }^{1} \mathrm{H}$ NMR $\left(400 \mathrm{MHz}, \mathrm{CDCl}_{3}\right.$, r.t. $)$
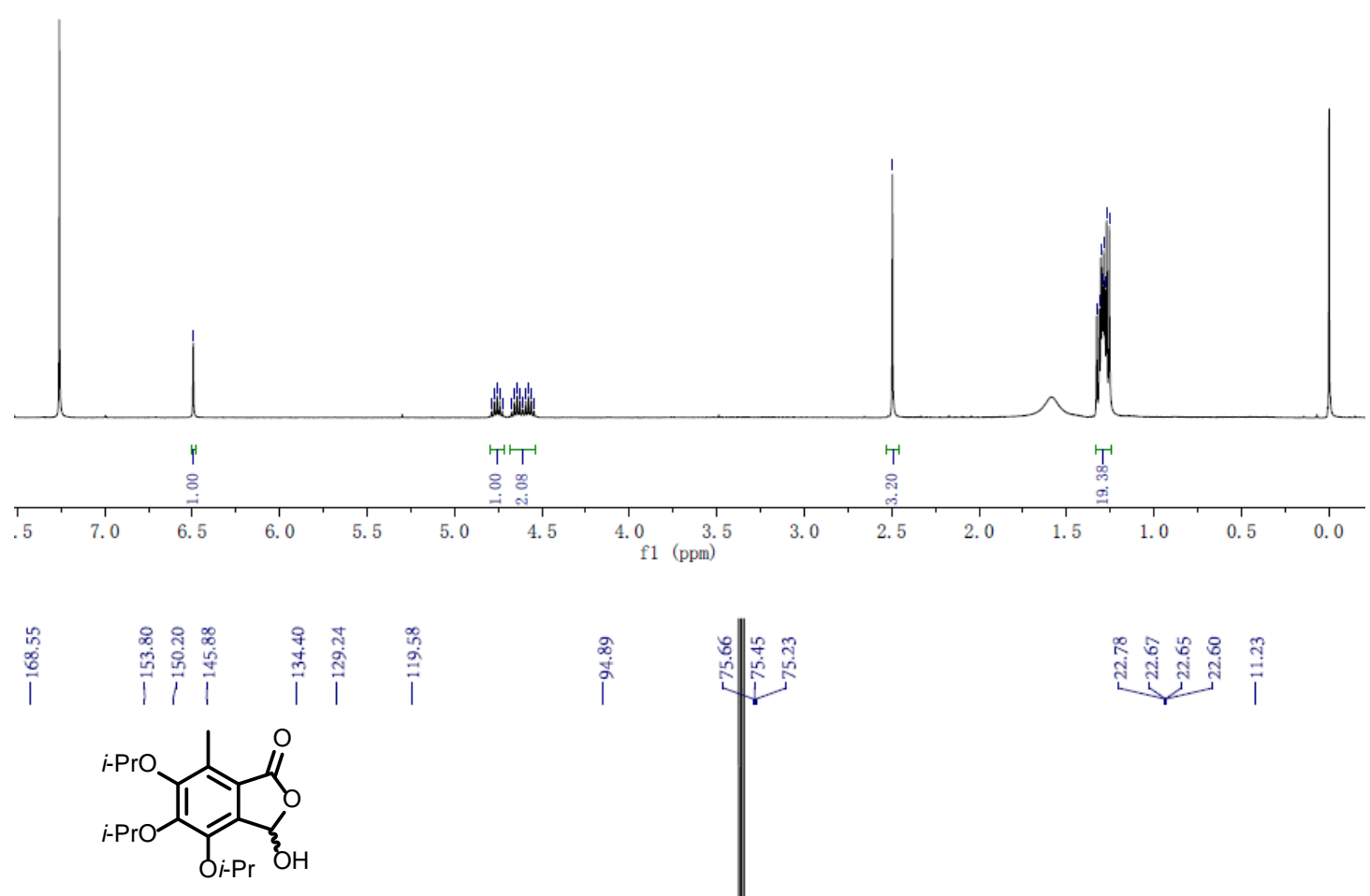

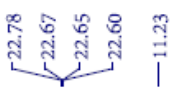

${ }^{13} \mathrm{C}\left\{{ }^{1} \mathrm{H}\right\}$ NMR $\left(101 \mathrm{MHz}, \mathrm{CDCl}_{3}\right.$, r.t. $)$

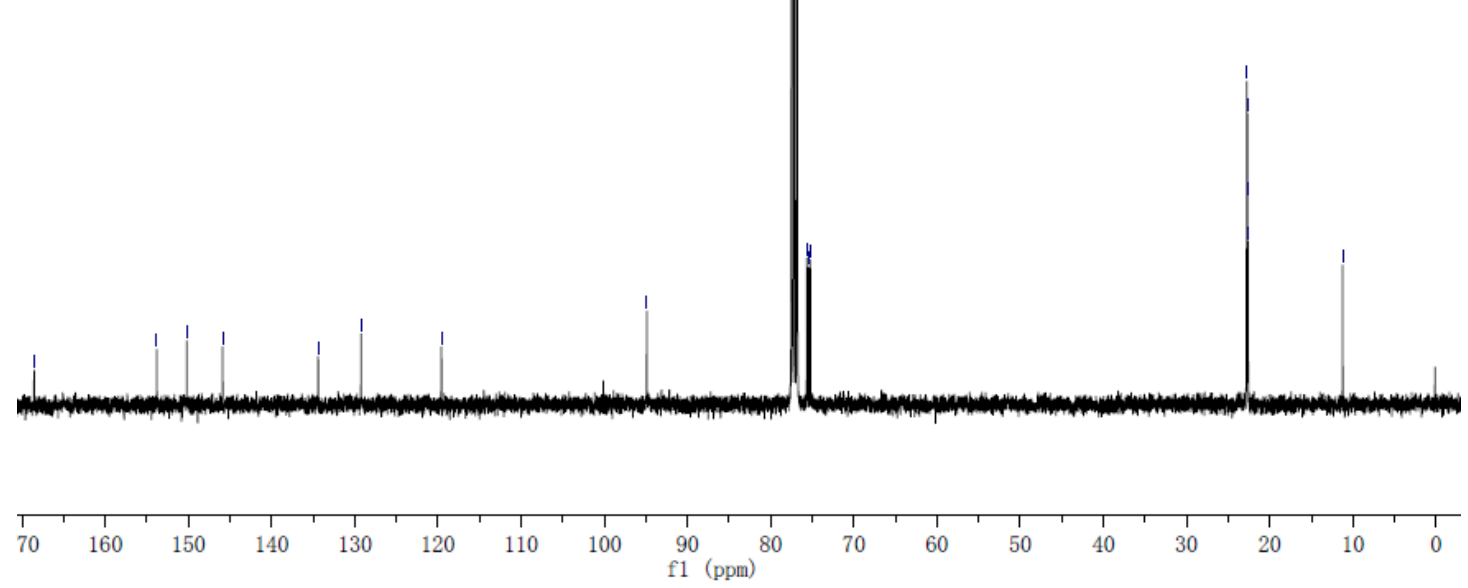




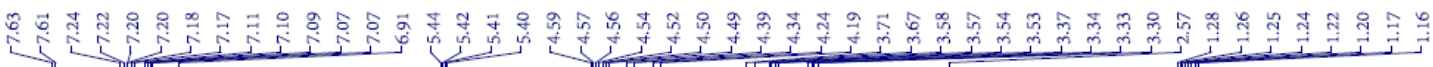

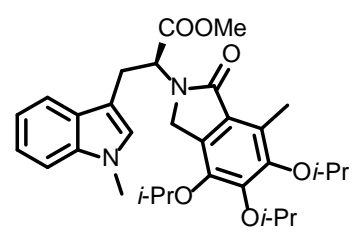

14

${ }^{1} \mathrm{H}$ NMR $\left(400 \mathrm{MHz}, \mathrm{CDCl}_{3}\right.$, r.t. $)$

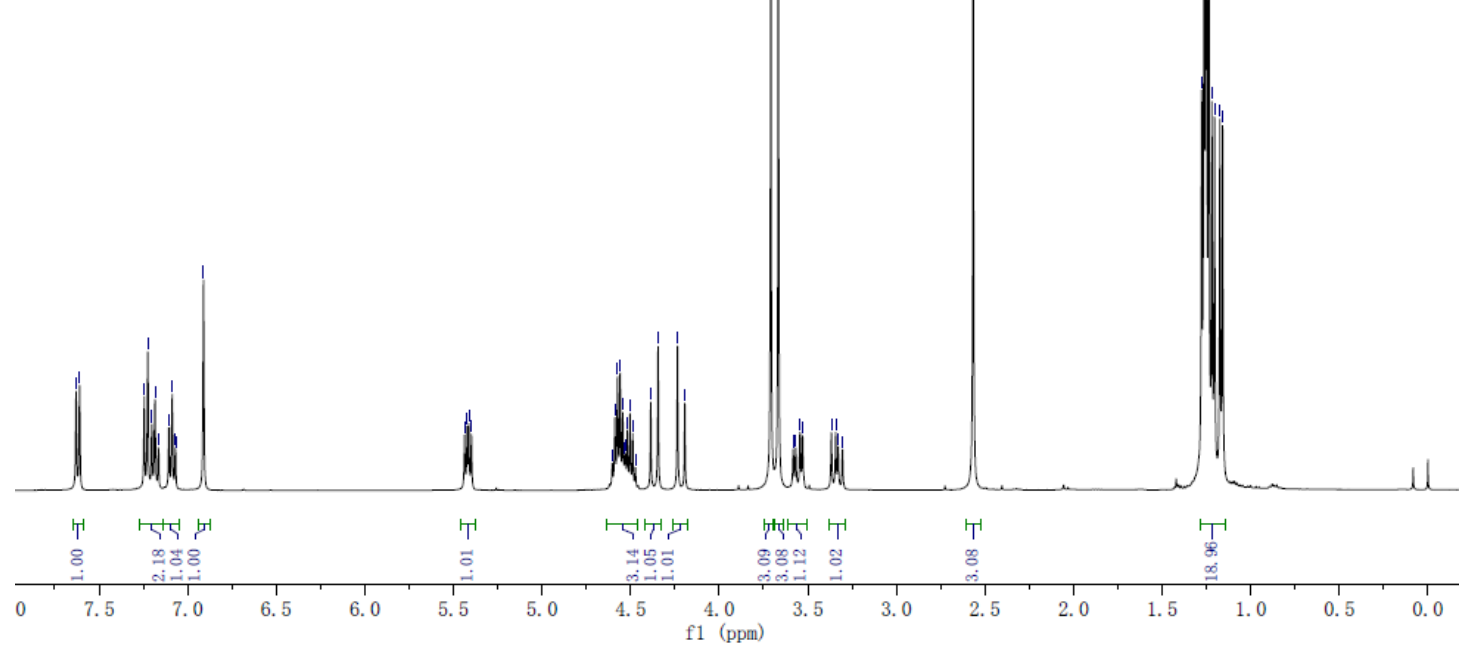

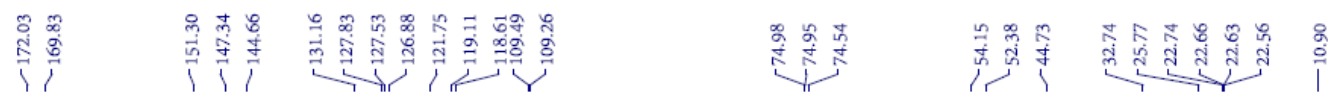

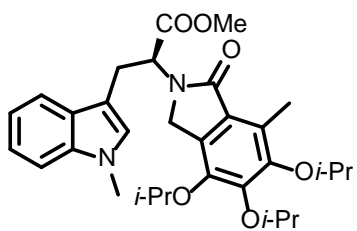

14

${ }^{13} \mathrm{C}\left\{{ }^{1} \mathrm{H}\right\}$ NMR $\left(101 \mathrm{MHz}, \mathrm{CDCl}_{3}\right.$, r.t.)

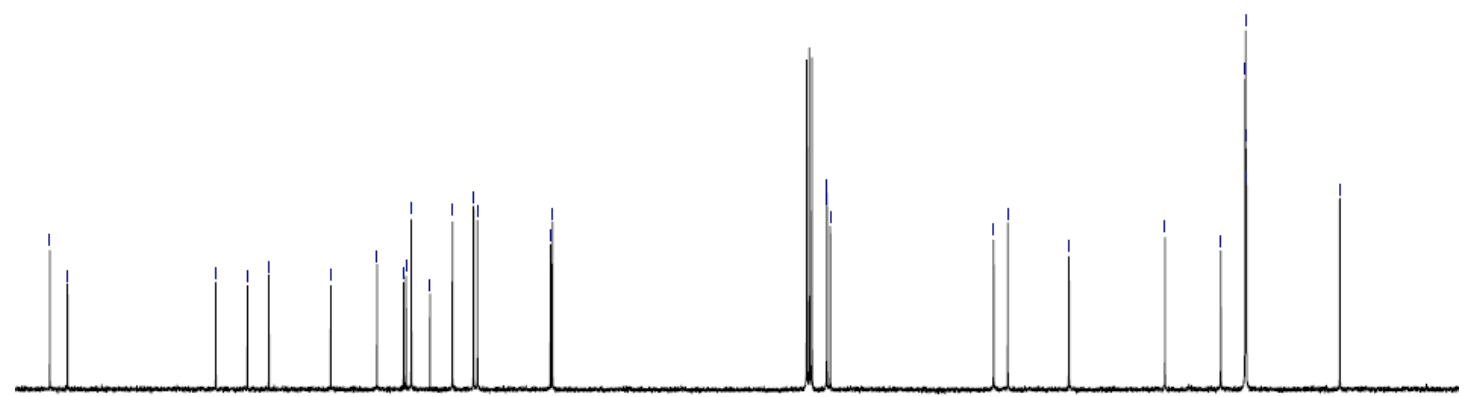

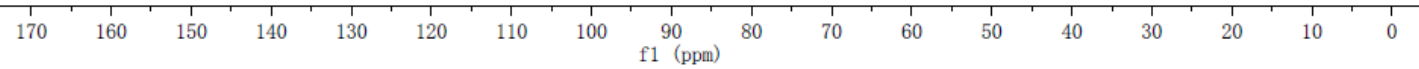




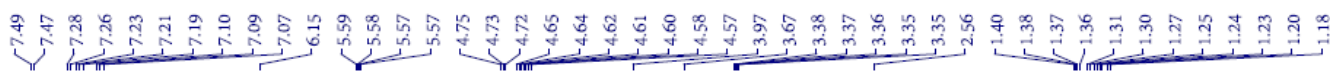

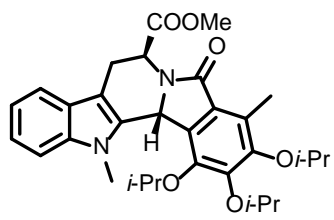

15

${ }^{1} \mathrm{H}$ NMR $\left(400 \mathrm{MHz}, \mathrm{CDCl}_{3}\right.$, r.t.)

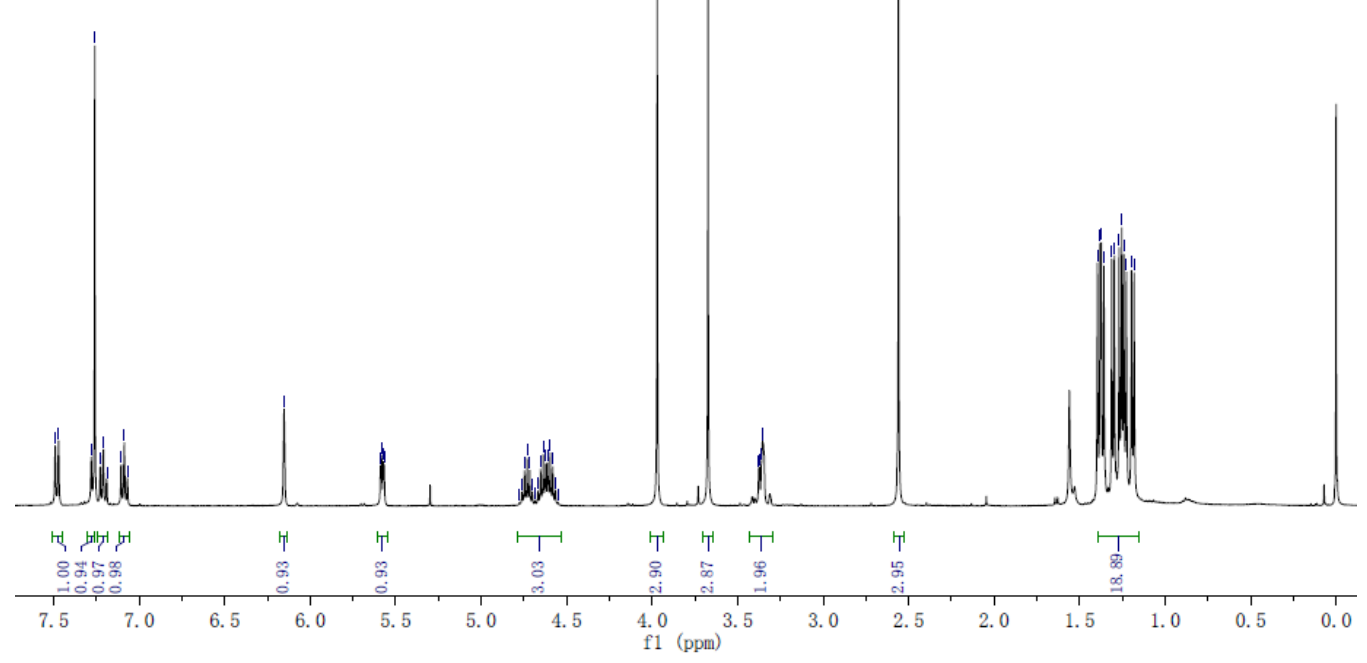

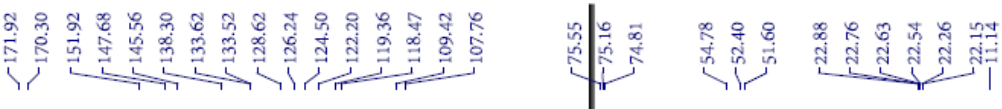

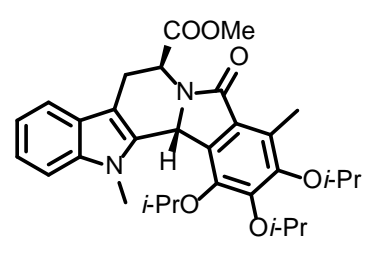

15

${ }^{13} \mathrm{C}\left\{{ }^{1} \mathrm{H}\right\}$ NMR $\left(101 \mathrm{MHz}, \mathrm{CDCl}_{3}\right.$, r.t. $)$

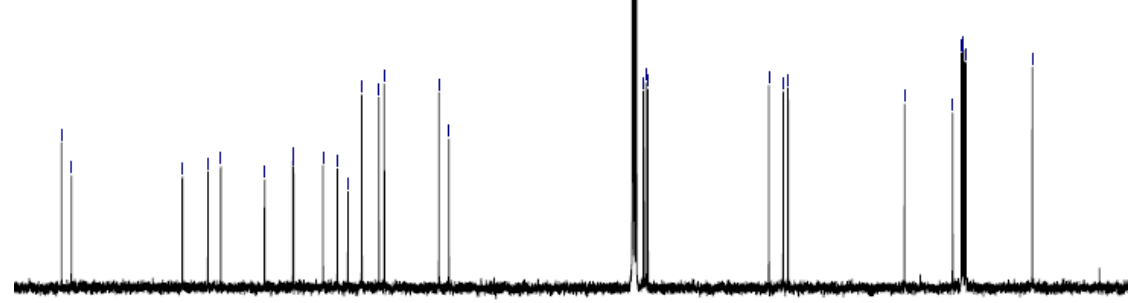

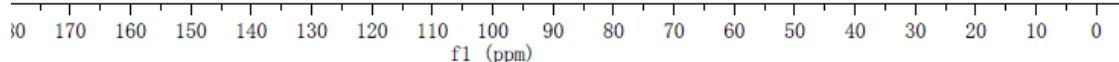




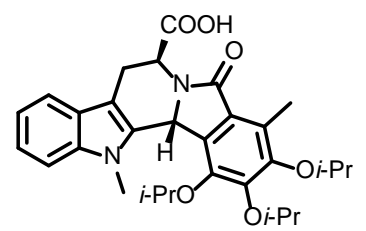

16

${ }^{1} \mathrm{H}$ NMR (400 MHz, $\mathrm{CDCl}_{3}$, r.t.)
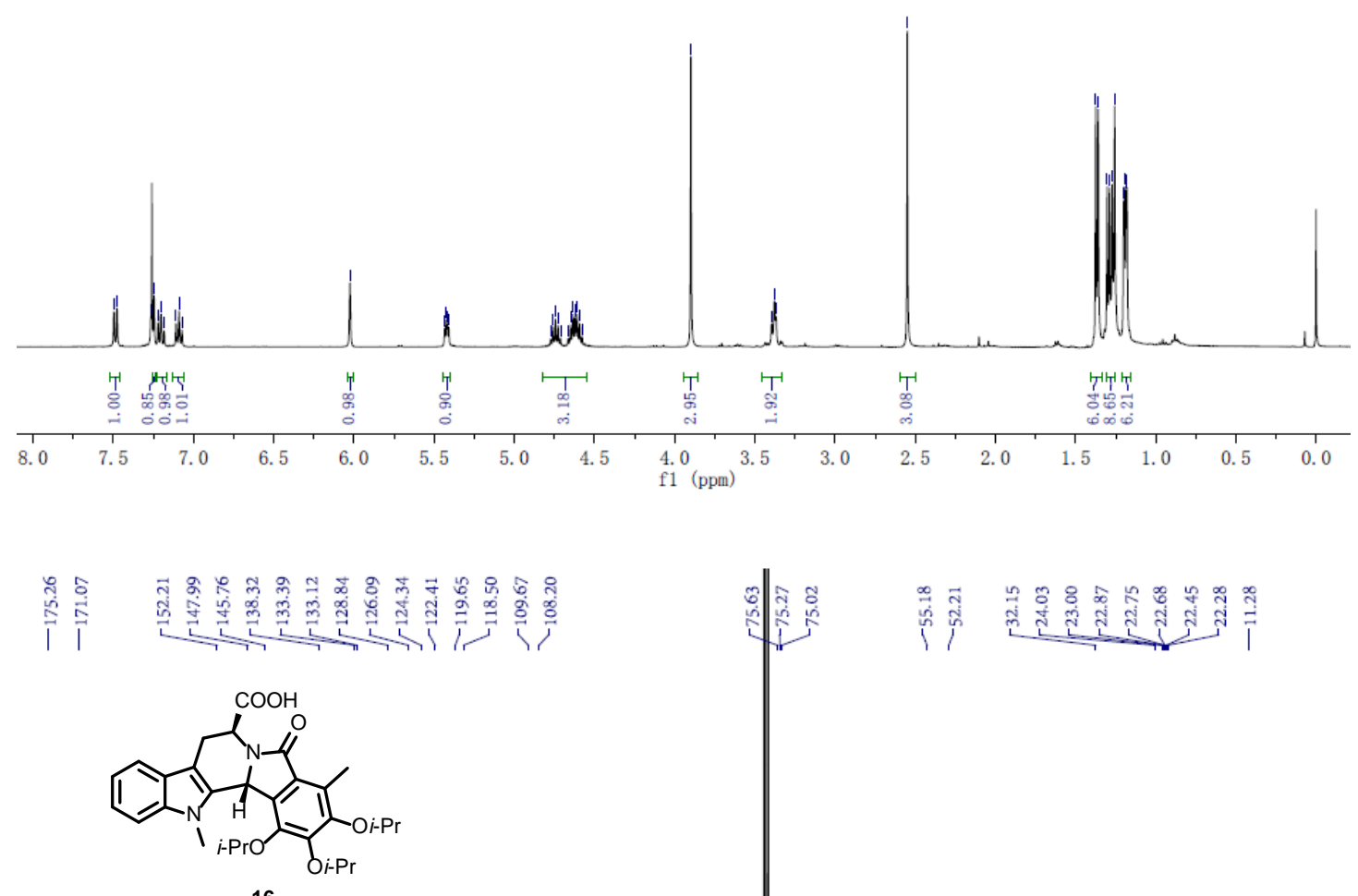

${ }^{13} \mathrm{C}\left\{{ }^{1} \mathrm{H}\right\}$ NMR (101 MHz, $\mathrm{CDCl}_{3}$, r.t. $)$
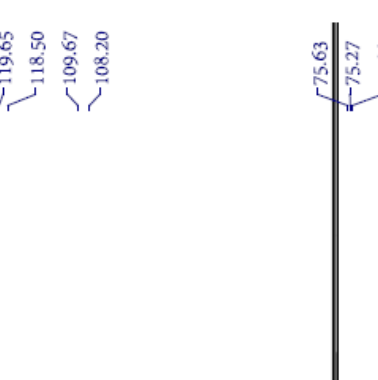

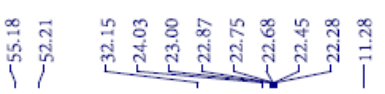

$r_{0}^{170} \quad 160 \quad 150 \quad 140 \quad 130$

1 (ppm) 


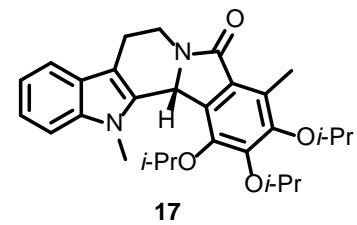

${ }^{1}{ }_{\mathrm{H}} \mathrm{NMR}\left(400 \mathrm{MHz}, \mathrm{CDCl}_{3}\right.$, r.t. $)$
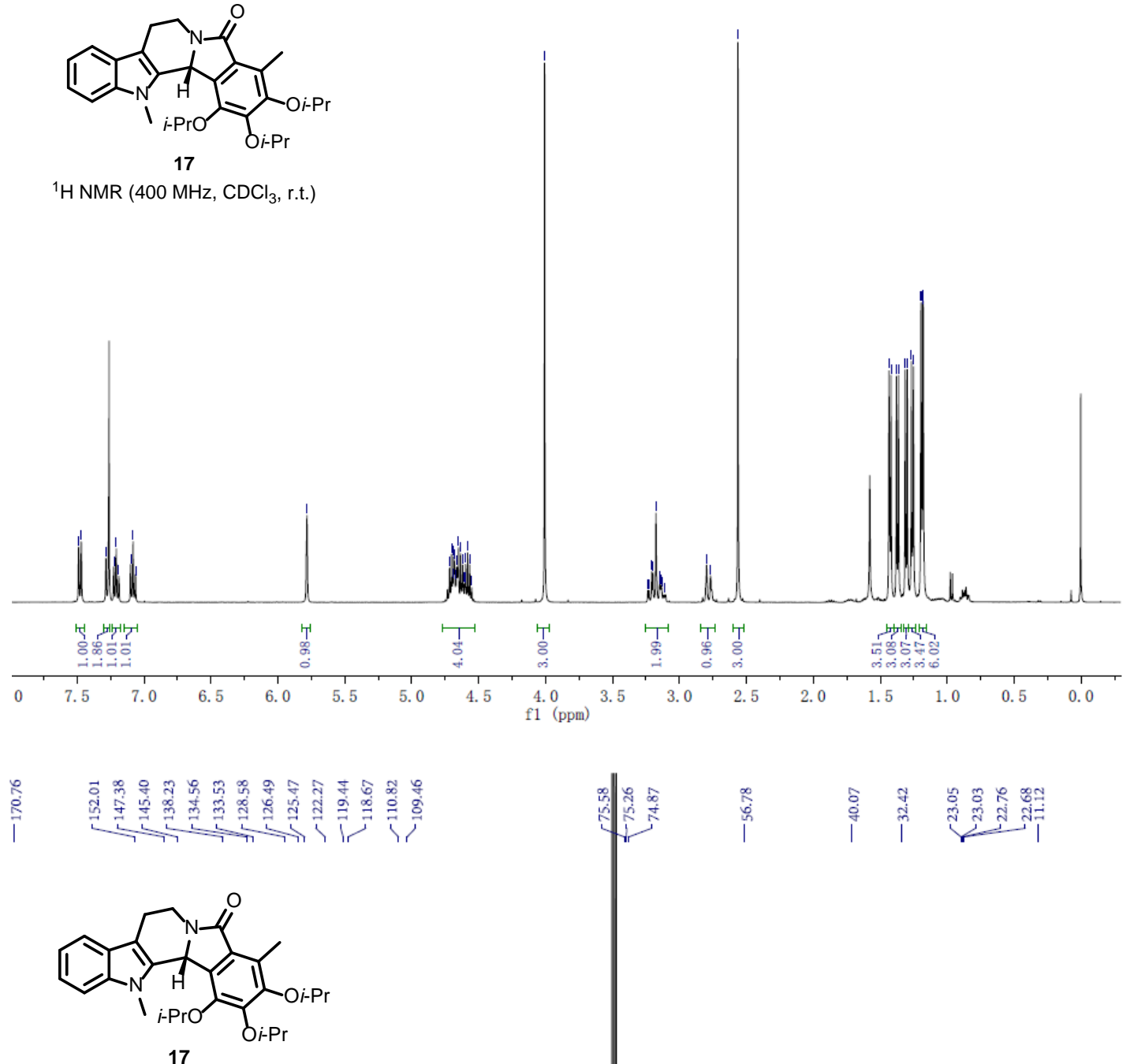

${ }^{13} \mathrm{C}\left\{{ }^{1} \mathrm{H}\right\}$ NMR $\left(101 \mathrm{MHz}, \mathrm{CDCl}_{3}\right.$, r.t. $)$ 


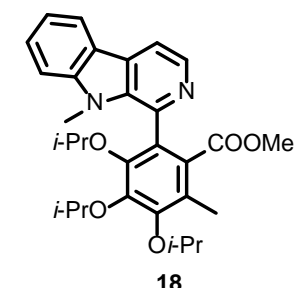

${ }^{1} \mathrm{H}$ NMR (400 MHz, $\mathrm{CDCl}_{3}$, r.t.)
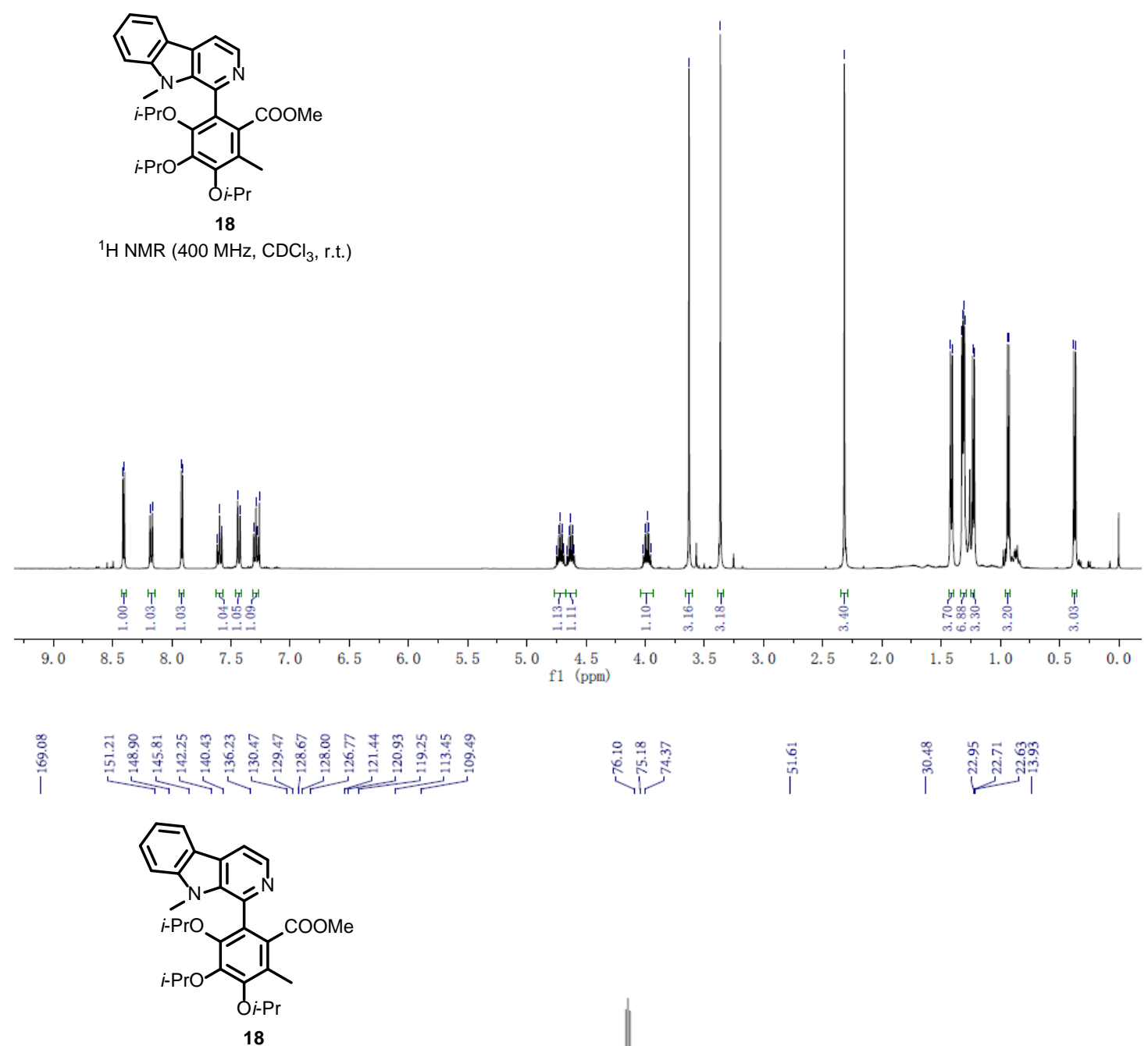

${ }^{13} \mathrm{C}\left\{{ }^{1} \mathrm{H}\right\}$ NMR (101 MHz, $\mathrm{CDCl}_{3}$, r.t. $)$ 


\section{Biological activity of chaetogline $F$}

The acetylcholinesterase (AChE)inhibitory assay was performed as reported. ${ }^{2}$

Table S10. Inhibitory activity of chaetogline F on Acetylcholinesterase.

\begin{tabular}{cc}
\hline Concentration $(\mu \mathrm{M})$ & The percent inhibition $(\%)^{*}$ \\
\hline 15.0 & $88.52 \pm 2.13$ \\
10.0 & $80.46 \pm 1.85$ \\
7.0 & $71.95 \pm 2.21$ \\
5.0 & $59.88 \pm 1.78$ \\
2.0 & $45.66 \pm 2.63$ \\
1.0 & $35.09 \pm 2.90$ \\
0.5 & $23.65 \pm 2.24$ \\
\hline
\end{tabular}

*mean $\pm \mathrm{SD},(\mathrm{n}=3)$

$\mathrm{IC}_{50}=2.26 \mu \mathrm{M}$

Figure S1. Dose-dependent inhibition curve of chaetogline F

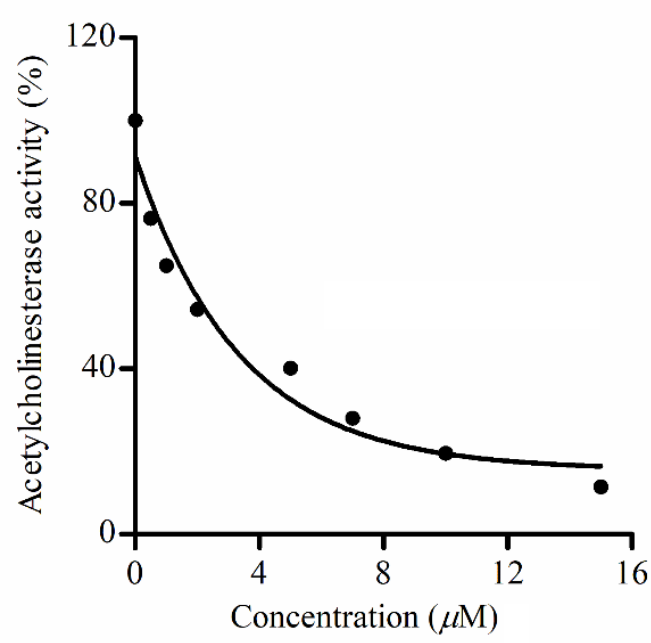




\section{References}

1. Yan, W.; Ge, H. M.; Wang, G.; Jiang, N.; Mei, Y. N.; Jiang, R.; Li, S.; Chen, C.; Jiao, R.; Xu, Q.; Seik, W.; Tan, R. X. Pictet-Spengler reaction-based biosynthetic machinery in fungi. Proc. Nat. Acad. Sci. USA. 2014, 111, 18138-18143.

2. Ge, H. M.; Zhu, C. H.; Shi, D. H.; Zhang, D. L.; Xie, D. Q.; Yang, J.; Seik, W.; Tan, R. X. Hopeahainol A: An Acetylcholinesterase Inhibitor from Hopea hainanensis. Chem. Eur. J, 2008, 14, 376-381. 